2018-6

\title{
Development of a Vortex Particle Code for the Modeling of Wake Interaction in Distributed Propulsion
}

\author{
Eduardo Alvarez \\ Brigham Young University, ealvarez@byu.edu \\ Andrew Ning \\ Brigham Young University, aning@byu.edu
}

Follow this and additional works at: https://scholarsarchive.byu.edu/facpub

Part of the Aerospace Engineering Commons

\section{Original Publication Citation}

Alvarez, E. J., and Ning, A., "Development of a Vortex Particle Code for the Modeling of Wake Interaction in Distributed Propulsion," AIAA Applied Aerodynamics Conference, Atlanta, GA, Jun. 2018. doi:10.2514/6.2018-3646

\section{BYU ScholarsArchive Citation}

Alvarez, Eduardo and Ning, Andrew, "Development of a Vortex Particle Code for the Modeling of Wake Interaction in Distributed Propulsion" (2018). Faculty Publications. 2116.

https://scholarsarchive.byu.edu/facpub/2116 


\title{
Development of a Vortex Particle Code for the Modeling of Wake Interaction in Distributed Propulsion
}

\author{
Eduardo J. Alvarez* and Andrew Ning ${ }^{\dagger}$ \\ Brigham Young University, Provo, Utah, 84602
}

\begin{abstract}
Recent developments in electric aircraft technology have enabled the use of distributed propulsion for the next generation of vertical lift vehicles. However, the ability to rapidly assess the performance of these design concepts, with sufficient fidelity, is a current weakness of this nascent industry. This paper explores the capacity of the viscous Vortex Particle Method (VPM) to model wake interactions found in distributed propulsion. The elements of the vortex particle method are summarized, and a new approach for the calculation of vortex stretching through the complex-step derivative approximation is presented. Preliminary validation is performed on vortex ring cases resembling the fundamental dynamics encountered in propeller wakes. Unsteady wake dynamics of individual propellers are successfully modeled, replicating the instabilities that lead to vortex breakdown as observed experimentally. Comparing the method with results from momentum theory, it is shown that VPM is consistent with theoretical values of near and far field induced velocities, and a notable feature is its ability to model near/far field transition. Furthermore, VPM is able to fully characterize induced velocities across the entire wake, from the stable region where momentum theory operates, through instability transition and eventual vortex breakdown. The simulation of a multirotor configuration of two tip-to-tip propellers is shown, displaying the capacity of VPM to model wake mixing. The results presented here are intermediate steps in the development of a mid-fidelity modeling tool for the early design stages of distributed-propulsion electric aircraft.
\end{abstract}

\section{Introduction}

Recent developments in electric aircraft technology have enabled the use of distributed propulsion for the next generation of vertical lift vehicles. However, distributed propulsion introduces aerodynamic interactions that are not well understood. One major challenge is that the use of many rotors in close proximity creates strong aerodynamic interactions between the rotors and lifting surfaces. In this study, we are particularly interested in the possible benefits and penalties of prop-on-prop interactions. High-fidelity computational fluid dynamics (CFD) and wind tunnel testing can be used to assess these interactions, but these approaches are too expensive to accommodate divergent design exploration. Current low-fidelity analysis methods are ill-equipped to assess the aerodynamic performance of novel multirotor configurations at the conceptual design level. Because this is an emerging field, established design guidelines and best practices do not exist. The ability to rapidly assess the performance of these design concepts, with sufficient fidelity, is a current weakness of this nascent industry.

One promising mid-fidelity approach for modeling complex wake interactions is the viscous Vortex Particle Method (VPM). This method is a first-principles-based approach for modeling unsteady dynamics, mixing, and viscous decay of wakes. Multiple vortex panel and filament methods have been developed for the modeling of free wakes, but experience shows that these methods are ill-conditioned for the modeling of complex dynamics. The requirement of preserving connectivity make traditional free-wake methods unable to model the mixing of wakes and viscous effects. On the other hand, the vortex particle method discretizes

\footnotetext{
*Graduate Student, Department of Mechanical Engineering, ealvarez@byu.edu.

${ }^{\dagger}$ Assistant Professor, Department of Mechanical Engineering, aning@byu.edu, AIAA Senior Member.
} 
the vorticity field into free particles, and solves the vorticity form of Navier-Stokes equations in a Lagrangian scheme. In past work, mid/high-fidelity simulations have been obtained through VPM at a relatively low computational cost. Berdowski ${ }^{1}$ used VPM to characterize the wake downstream of an actuator-disk wind turbine model. Calabretta ${ }^{2}$ used VPM to model prop-on-wing interaction between an actuator-disk propeller and a paneled wing. Stock et al. ${ }^{3}$ modeled an isolated rotor in forward flight in a hybrid CFD-VPM approach. He et al. ${ }^{4-6}$ used VPM to study the interaction between coaxial rotors, coupling a lifting-line wing to model rotor-on-wing interaction, and a hybrid VPM-CFD solver to characterize rotor-on-fuselage interaction of a full rotorcraft. Most recently, Ho and Yeo ${ }^{7}$ coupled He and Zhao's VPM ${ }^{4}$ with the US Army's suite of analysis tools to model forward flight and validate predicted unsteady loads with experimental tiltrotor data.

VPM has been successfully applied to a variety of wake-dominated flow, ${ }^{8-16}$ especially the cases of coaxial rotors ${ }^{17,18}$ and rotorcraft forward flight. ${ }^{19-23}$ However, it has not yet been applied to the coplanar multirotor configuration. This paper explores the capacity of the viscous vortex particle method to model wake interactions encountered in distributed propulsion. A propeller model is developed coupling blade element momentum theory and the vortex lattice method with VPM. In Section II the modeling methodology is presented, summarizing the principles of the vortex particle as implemented in our VPM code. The Fast Multipole Method is applied for accelerating the computation of particle-to-particle interaction, and an approach for the evaluation of vortex stretching through complex-step derivative approximation is presented. The fundamental phenomena observed in propeller wakes is analogous to the dynamics of vortex rings, hence, in Section III preliminary validation is presented through the modeling of vortex-ring dynamics. Finally, in Section IV the unsteady wake dynamics of an APC 10x7 propeller are simulated in individual and multirotor configurations.

\section{Modeling Methodology}

\section{II.A. Viscous Vortex Particle Method}

In the subsequent sections the fundamental principles of the vortex particle method are reviewed. In Section II.A.1, the governing equations of the method are derived from the Navier-Stokes equations. Assuming incompressible flow, the velocity field outside the boundary layer is calculated as a vector-potential flow dominated by vorticity. The vorticity field is discretized and regularized in a Lagrangian scheme as vortex particles, as expounded in Section II.A.2. Viscous effects are modeled through the Particle Strength Exchange scheme summarized in Section II.A.3. In order to apply the VPM to practical cases requiring large number of particles, the Fast Multipole Method is implemented as explained in Section II.A.4, and an approach for the calculation of vortex stretching using complex-step derivative approximation is developed in Section II.A.5.

\section{II.A.1. Governing Equations}

From the Stokes' assumptions for a Newtonian, incompressible fluid with constant viscosity, the conservation of momentum in a differential fluid element is governed by the well-known Navier-Stokes equations:

$$
\frac{\partial \mathbf{u}}{\partial t}+(\mathbf{u} \cdot \nabla) \mathbf{u}=-\frac{1}{\rho} \nabla p+\nu \nabla^{2} \mathbf{u}
$$

Using the identity $(\mathbf{u} \cdot \nabla) \mathbf{u}=\frac{1}{2} \nabla(\mathbf{u} \cdot \mathbf{u})+(\nabla \times \mathbf{u}) \times \mathbf{u}$, this equation can then be rewritten as

$$
\frac{\partial \mathbf{u}}{\partial t}+\boldsymbol{\omega} \times \mathbf{u}=-\nabla\left(\frac{p}{\rho}+\frac{\mathbf{u} \cdot \mathbf{u}}{2}\right)+\nu \nabla^{2} \mathbf{u}
$$

where $\mathbf{u}(\mathbf{x}, t)$ is the velocity field, $\boldsymbol{\omega}(\mathbf{x}, t)=\nabla \times \mathbf{u}(\mathbf{x}, t)$ is the vorticity field, $p(\mathbf{x}, t)$ is the pressure field, and $\rho$ and $\nu$ are the density and kinematic viscosity of the fluid, respectively. Taking the curl over Eq. (2), and using the identity $\nabla \times\left(\nabla^{2} \mathbf{u}\right)=\nabla^{2}(\nabla \times \mathbf{u})$, the momentum equation is then transformed into its vorticty form

$$
\frac{\partial \boldsymbol{\omega}}{\partial t}+\nabla \times(\boldsymbol{\omega} \times \mathbf{u})=\nu \nabla^{2} \boldsymbol{\omega}
$$

The second term in the left hand side can be expanded as $\nabla \times(\boldsymbol{\omega} \times \mathbf{u})=\boldsymbol{\omega}(\nabla \cdot \mathbf{u})-\mathbf{u}(\nabla \cdot \boldsymbol{\omega})+(\mathbf{u} \cdot \nabla) \boldsymbol{\omega}-(\boldsymbol{\omega} \cdot \nabla) \mathbf{u}$. From the continuity equation of an incompressible fluid $(\nabla \cdot \mathbf{u}=0)$ and the definition of vorticity $(\boldsymbol{\omega}=\nabla \times \mathbf{u})$, 
both $\mathbf{u}$ and $\boldsymbol{\omega}$ are found to be divergence-free fields, which further reduces that term to $\nabla \times(\boldsymbol{\omega} \times \mathbf{u})=$ $(\mathbf{u} \cdot \nabla) \boldsymbol{\omega}-(\boldsymbol{\omega} \cdot \nabla) \mathbf{u}$, and Eq. (3) becomes

$$
\frac{D \boldsymbol{\omega}}{D t}=(\boldsymbol{\omega} \cdot \nabla) \mathbf{u}+\nu \nabla^{2} \boldsymbol{\omega}
$$

which describes the evolution of the vorticity field.

The material derivative expressed in Eq. (4), and the material-conservative nature of the vorticity field makes $\boldsymbol{\omega}$ especially fit for a Lagrangian description. In order to do so, the field is discretized into vortex particles as

$$
\begin{aligned}
\boldsymbol{\omega}(\mathbf{x}, t) & \approx \sum_{p} \boldsymbol{\omega}_{p}(t) \operatorname{vol}_{p} \delta\left(\mathbf{x}-\mathbf{x}_{p}(t)\right) \\
& \approx \sum_{p} \boldsymbol{\Gamma}_{p}(t) \delta\left(\mathbf{x}-\mathbf{x}_{p}(t)\right)
\end{aligned}
$$

where $\boldsymbol{\omega}_{p}$ is the vorticity associated to the $p$-th particle of volume $\operatorname{vol}_{p}$, and $\boldsymbol{\Gamma}_{p} \equiv \int_{\text {vol }_{p}} \boldsymbol{\omega} \mathrm{d} V$ is its vectorial circulation, or vectorial vortex strength. Each particle is a fluid element that travels with the local velocity as in Eq. (7), where $\mathbf{x}_{p}$ is the position of the $p$-th particle. Substituting Eq. (6) in Eq. (4), it is obtained that the vectorial circulation of each particle evolves as in Eq. (8), where the first right-hand-side term is the vortex stretching, while the second represent a scheme for modeling the viscous diffusion $\nu \nabla^{2} \omega$. Thus, Eqs. (7) and (8) become the governing equations for the evolution of vorticity-governed flow in a Lagrangian scheme.

$$
\begin{aligned}
\frac{\mathrm{d}}{\mathrm{d} t} \mathbf{x}_{p}(t) & =\mathbf{u}\left(\mathbf{x}_{p}(t), t\right) \\
\frac{\mathrm{d}}{\mathrm{d} t} \boldsymbol{\Gamma}_{p}(t) & =\left(\boldsymbol{\Gamma}_{p}(t) \cdot \nabla\right) \mathbf{u}\left(\mathbf{x}_{p}(t), t\right)+\left.\frac{\mathrm{d}}{\mathrm{d} t} \boldsymbol{\Gamma}_{p}(t)\right|_{\text {visc }}
\end{aligned}
$$

\section{II.A.2. Kernel Regularization}

We now describe the calculation of the velocity field $\mathbf{u}$ used in the governing equations (7) and (8). By the Helmholtz Decomposition Theorem, the divergence-free field $\mathbf{u}$ can be decomposed as $\mathbf{u}=\mathbf{u}_{\infty}+\mathbf{u}_{\boldsymbol{\omega}}+$ $\mathbf{u}_{\phi}=\mathbf{u}_{\infty}+\nabla \times \boldsymbol{\psi}+\nabla \phi$, with $\mathbf{u}_{\infty}(t)$ a uniform free-stream field, $\mathbf{u}_{\boldsymbol{\omega}}$ a component defined by some vector potential field $\boldsymbol{\psi}$ as $\mathbf{u}_{\boldsymbol{\omega}}(\mathbf{x}, t)=\nabla \times \boldsymbol{\psi}(\mathbf{x}, t)$, and $\mathbf{u}_{\phi}$ a component defined by some scalar potential field $\phi$ as $\mathbf{u}_{\phi}(\mathbf{x}, t)=\nabla \phi(\mathbf{x}, t)$. For the purpose of our study, $\mathbf{u}_{\infty}$ and $\mathbf{u}_{\phi}$ represent the freestream and the velocity induced by a lifting surface, respectively, which are calculated independently from the VPM; meanwhile $\mathbf{u}_{\boldsymbol{\omega}}=\nabla \times \boldsymbol{\psi}$ is the velocity induced by the particle field. We are interested in determining the vortex-particle induced velocity $\mathbf{u}_{\boldsymbol{\omega}}$, so in this section both scalar potential and uniform components will be ignored, with $\mathbf{u}$ becoming $\mathbf{u}=\mathbf{u}_{\boldsymbol{\omega}}$, and we will develop an expression for the vector-potential field $\boldsymbol{\psi}$.

Having defined the vorticity field as $\boldsymbol{\omega}=\nabla \times \mathbf{u}$, we have

$$
\begin{aligned}
\boldsymbol{\omega} & =\nabla \times(\nabla \times \boldsymbol{\psi}) \\
& =\nabla(\nabla \cdot \boldsymbol{\psi})-\nabla^{2} \boldsymbol{\psi},
\end{aligned}
$$

where the first term in the right side of Eq. (10) is nullified after applying a gauge transform [24, Sec. 6.3], defining the Poisson's equation

$$
\nabla^{2} \boldsymbol{\psi}=-\boldsymbol{\omega}
$$

The fundamental solution of Poisson's equation of the form $\nabla^{2} u(\mathbf{x})=f(\mathbf{x})$ is given by Green's function $G\left(\mathbf{x}, \mathbf{x}^{\prime}\right)$ such that

$$
u(\mathbf{x})=\int_{V} G\left(\mathbf{x}, \mathbf{x}^{\prime}\right) f\left(\mathbf{x}^{\prime}\right) d \mathbf{x}^{\prime}
$$




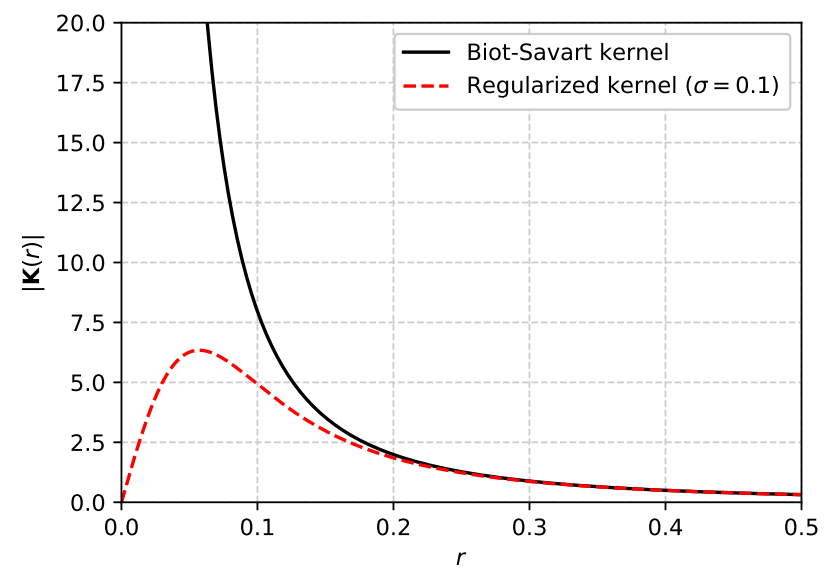

Figure 1: Comparison of the singular Biot-Savart kernel (Eq. (19)) to the regularized kernel in Eq. (20).

where $G\left(\mathbf{x}, \mathbf{x}^{\prime}\right)$ describes the influence that a point source located at $\mathbf{x}^{\prime}$ exerts at a point $\mathbf{x}$, and $\nabla^{2} G\left(\mathbf{x}, \mathbf{x}^{\prime}\right)=$ $\delta\left(\mathbf{x}-\mathbf{x}^{\prime}\right)$. Applying this to Eq. (11), we have

$$
\psi(\mathbf{x}, t)=\int_{V} G\left(\mathbf{x}, \mathbf{x}^{\prime}\right) \boldsymbol{\omega}\left(\mathbf{x}^{\prime}, t\right) d \mathbf{x}^{\prime} .
$$

Using the particle approximation $\boldsymbol{\omega}(\mathbf{x}, t) \approx \sum_{p} \boldsymbol{\Gamma}_{p}(t) \delta\left(\mathbf{x}-\mathbf{x}_{p}(t)\right)$, the integral in Eq. (13) becomes

$$
\psi(\mathbf{x}, t)=\sum_{p} G\left(\mathbf{x}-\mathbf{x}_{p}(t)\right) \boldsymbol{\Gamma}_{p}(t)
$$

and applying the curl identity $\nabla \times(\phi \mathbf{A})=(\nabla \phi) \times \mathbf{A}+\phi(\nabla \times \mathbf{A})$, an expression for the velocity field is obtained as

$$
\begin{aligned}
\mathbf{u}(\mathbf{x}, t) & =\nabla \times \boldsymbol{\psi}(\mathbf{x}, t) \\
& =\sum_{p}[\nabla G\left(\mathbf{x}-\mathbf{x}_{p}(t)\right) \times \boldsymbol{\Gamma}_{p}(t)+G\left(\mathbf{x}-\mathbf{x}_{p}(t)\right) \overbrace{\nabla \times \boldsymbol{\Gamma}_{p}(t)}^{0}] \\
& =\sum_{p} \nabla G\left(\mathbf{x}-\mathbf{x}_{p}(t)\right) \times \mathbf{\Gamma}_{p}(t)
\end{aligned}
$$

or

$$
\mathbf{u}(\mathbf{x}, t)=\sum_{p} \mathbf{K}\left(\mathbf{x}-\mathbf{x}_{p}(t)\right) \times \boldsymbol{\Gamma}_{p}(t) \quad, \text { with } \quad \mathbf{K}=\nabla G .
$$

Employing the three-dimensional Newtonian kernel (or Biot-Savart kernel), $G\left(\mathbf{x}, \mathbf{x}^{\prime}\right)=\frac{1}{4 \pi\left\|\mathbf{x}-\mathbf{x}^{\prime}\right\|}$, the velocity field is obtained as

$$
\mathbf{u}(\mathbf{x}, t)=-\frac{1}{4 \pi} \sum_{p} \frac{\mathbf{x}-\mathbf{x}_{p}}{\left\|\mathbf{x}-\mathbf{x}_{p}\right\|^{3}} \times \boldsymbol{\Gamma}_{p}(t)
$$

However, the kernel $\mathbf{K}\left(\mathbf{x}-\mathbf{x}_{p}\right)=-\frac{1}{4 \pi} \frac{\mathbf{x}-\mathbf{x}_{p}}{\left\|\mathbf{x}-\mathbf{x}_{p}\right\|^{3}}$, or $\|\mathbf{K}(r)\|=\frac{1}{4 \pi r^{2}}$, has a singularity as $\|\mathbf{K}(r \rightarrow 0)\| \rightarrow \infty$ (shown in Figure 1); hence, this kernel is often avoided in vortex particle methods. In order to regularize this singularity, multiple alternative kernels have been proposed in the literature meeting different algebraic properties. The procedure for regularization is to define the vortex particle as a vortex "blob" with a smoothing radius $\sigma$ where its influence decays as the distance to its core approaches zero. In the code developed for this study, the high-order algebraic kernel proposed by Winckelmans and Leonard ${ }^{25}$ will be 
used as given in Eq. (20). Figure 1 shows a comparison of this regularized kernel to the singular Biot-Savart kernel.

$$
\mathbf{K}_{\sigma}(r)=-\frac{r\left[\left(\frac{r}{\sigma}\right)^{2}+5 / 2\right]}{4 \pi \sigma^{3}\left[\left(\frac{r}{\sigma}\right)^{2}+1\right]^{5 / 2}} \hat{r}
$$

\section{II.A.3. Viscous Diffusion}

Viscous effects are modeled in the right-hand-side of Eq. (8) through the Particle Strength Exchange (PSE) scheme $^{25,26}$ as summarized here. Degond and Mas-Gallic ${ }^{27}$ showed that the Laplacian of the form $\nu \nabla^{2} \boldsymbol{\omega}$ can be approximated by an integral operator as

$$
\nu \nabla^{2} \boldsymbol{\omega}(\mathbf{x}) \approx \frac{2 \nu}{\sigma^{2}} \int(\boldsymbol{\omega}(\mathbf{y})-\boldsymbol{\omega}(\mathbf{x})) \eta_{\sigma}(\mathbf{x}-\mathbf{y}) \mathrm{d} \mathbf{y}
$$

with $\zeta$ a smoothing function of radial symmetry, $\eta(\rho)=-\frac{1}{\rho} \frac{\mathrm{d}}{\mathrm{d} \rho} \zeta(\rho), \eta_{\sigma}(\mathbf{x})=\frac{\eta(\|\mathbf{x}\| / \sigma)}{\sigma^{3}}$, and $\sigma$ the smoothing radius.

Discretizing space into particles of volume $\operatorname{vol}_{p}$ and positions $\mathbf{x}_{p}$, the integral of a field $f$ can be approximated by the quadrature formula

$$
\int f(\mathbf{x}) \mathrm{d} \mathbf{x} \approx \sum_{p} \operatorname{vol}_{p} f\left(\mathbf{x}_{p}\right) .
$$

Since the vorticity field $\boldsymbol{\omega}$ is discretized by the vortex particle field as

$$
\begin{aligned}
\boldsymbol{\omega}(\mathbf{x}) & \approx \sum_{p} \boldsymbol{\omega}_{p} \operatorname{vol}_{p} \delta\left(\mathbf{x}-\mathbf{x}_{p}\right) \\
& \approx \sum_{p} \boldsymbol{\Gamma}_{p} \delta\left(\mathbf{x}-\mathbf{x}_{p}\right)
\end{aligned}
$$

the integral operator in Eq. (21) can be calculated by applying the quadrature approximation on the vortex particle field with $f_{\mathbf{x}}(\mathbf{y})=(\boldsymbol{\omega}(\mathbf{y})-\boldsymbol{\omega}(\mathbf{x})) \eta_{\sigma}(\mathbf{x}-\mathbf{y})$. Evaluating the viscous diffusion term at a particle position $\mathbf{x}_{p}$, we have

$$
\begin{aligned}
\nu \nabla^{2} \boldsymbol{\omega}\left(\mathbf{x}_{p}\right) & \approx \frac{2 \nu}{\sigma^{2}} \int f_{\mathbf{x}_{p}}(\mathbf{y}) \mathrm{d} \mathbf{y} \\
& \approx \frac{2 \nu}{\sigma^{2}} \sum_{q}\left(\operatorname{vol}_{q} \boldsymbol{\omega}_{q}-\operatorname{vol}_{q} \boldsymbol{\omega}_{p}\right) \eta_{\sigma}\left(\mathbf{x}_{p}-\mathbf{x}_{q}\right)
\end{aligned}
$$

Multiplying Eq. (26) by $\operatorname{vol}_{p}$ and applying the vortex particle discretization $\boldsymbol{\Gamma}_{p}=\int_{\operatorname{vol}_{p}} \boldsymbol{\omega} \mathrm{d} V$, viscous diffusion effects are then approximated as

$$
\left.\frac{\mathrm{d}}{\mathrm{d} t} \boldsymbol{\Gamma}_{p}\right|_{\text {visc }}=\frac{2 \nu}{\sigma^{2}} \sum_{q}\left(\operatorname{vol}_{p} \boldsymbol{\Gamma}_{q}-\operatorname{vol}_{q} \boldsymbol{\Gamma}_{p}\right) \eta_{\sigma}\left(\mathbf{x}_{p}-\mathbf{x}_{q}\right) .
$$

Finally, applying Winckelmann's high-order regularizing core ${ }^{25}$ we have

$$
\begin{aligned}
& \eta_{\sigma}(\mathbf{x})=\frac{1}{8 \pi \sigma^{3}} \frac{105}{\left[\left(\frac{\|\mathbf{x}\|}{\sigma}\right)^{2}+1\right]^{9 / 2}} \\
& \left.\Rightarrow \frac{\mathrm{d}}{\mathrm{d} t} \boldsymbol{\Gamma}_{p}\right|_{v i s c}=\frac{105 \nu}{4 \pi \sigma^{5}} \sum_{q} \frac{\operatorname{vol}_{p} \boldsymbol{\Gamma}_{q}-\operatorname{vol}_{q} \boldsymbol{\Gamma}_{p}}{\left[\left(\frac{\left\|\mathbf{x}_{p}-\mathbf{x}_{q}\right\|}{\sigma}\right)^{2}+1\right]^{9 / 2}}
\end{aligned}
$$

In our code we implement the modification that He and Zhao ${ }^{4}$ suggest, which uses a modified smoothing parameter $\sigma_{i j}=\sqrt{\frac{\sigma_{i}^{2}+\sigma_{j}^{2}}{2}}$ that makes the PSE scheme symmetric such that the vortex strength lost by one particle is the same than that gained by the other, leading to a conservation of total strength. 


\section{II.A.4. Fast Multipole Method}

As given by Eq. (18), the computation of velocity at an arbitrary position is carried by summing over all vortex particles in the computational domain, and since the velocity at each particle is required for the governing equations, this method has a computational complexity of $\mathcal{O}\left(N^{2}\right)$ at each time step, where $N$ is the number of particles. Even though this scheme is conceptually simple, the direct calculation of particle interactions becomes computationally unfeasible as the number of particles escalates. This problem, termed the $N$-body problem, is known across all disciplines where the computation of pairwise interaction of particles is required, as in celestial motion, electrically charged particles, and even some models in economics. An alternative approach to the direct calculation of particle-to-particle interactions is the clustering of particles and approximation of direct interactions between well-separated particles with the interactions between clusters. A popular and simple implementation of this concept is the Treecode method ${ }^{28}$ that reduces the computational effort to $\mathcal{O}(N \log (N))$. An even more efficient implementation is the Fast Multipole Method (FMM) introduced by Greengard and Rokhlin. ${ }^{29}$ Selected as one of the top ten algorithms of the 20th century, FMM reduces the original $N$-body problem $\mathcal{O}\left(N^{2}\right)$ to a linear problem $\mathcal{O}(N)$; however, this method is often avoided due to its considerable conceptual and implementation complexity.

The FMM approximates pairwise interactions of particles of the form

$$
\Phi\left(\mathbf{x}_{j}\right)=\sum_{\substack{i=1 \\ i \neq j}}^{N} \frac{q_{i}}{\left\|\mathbf{x}_{j}-\mathbf{x}_{i}\right\|}
$$

through the multipole expansion

$$
\Phi(r, \theta, \phi) \approx \sum_{n=0}^{p} \sum_{m=-n}^{n} \frac{M_{n}^{m}}{r^{n+1}} Y_{n}^{m}(\theta, \phi) \quad, \text { with } \quad M_{n}^{m}=\sum_{i=1}^{N} q_{i} \rho_{i}^{n} Y_{n}^{-m}\left(\alpha_{i}, \beta_{i}\right),
$$

and the local expansion

$$
\Phi(r, \theta, \phi) \approx \sum_{n=0}^{p} \sum_{m=-n}^{n} L_{n}^{m} r^{n} Y_{n}^{m}(\theta, \phi) \quad, \text { with } \quad L_{n}^{m}=\sum_{i=1}^{N} q_{i} \rho_{i}^{n} Y_{n}^{-m}\left(\alpha_{i}, \beta_{i}\right),
$$

where - following the nomenclature used by Greengard ${ }^{30}-, Y_{n}^{m}(\theta, \phi)$ are the spherical harmonic functions, $(r, \theta, \phi)$ are the spherical coordinates of the target particle about the center of expansion, and $\left(\rho_{i}, \theta_{i}, \phi_{i}\right)$ are the coordinates of the $i$-th source particle. In this paper the authors will focus on the contributions

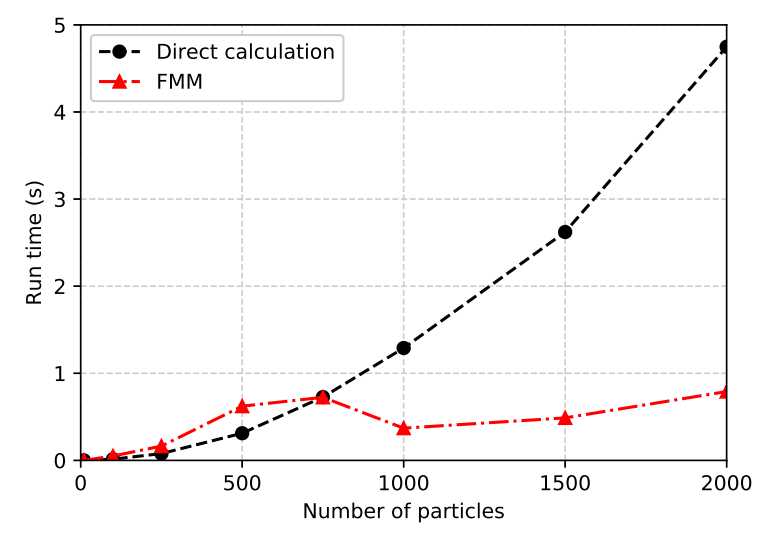

Figure 2: Performance comparison between direct calculation of particle-to-particle interactions to Fast Multipole Method (ExaFMM) on an expansion order $p=10$, and a maximum of 4 particles per cluster.

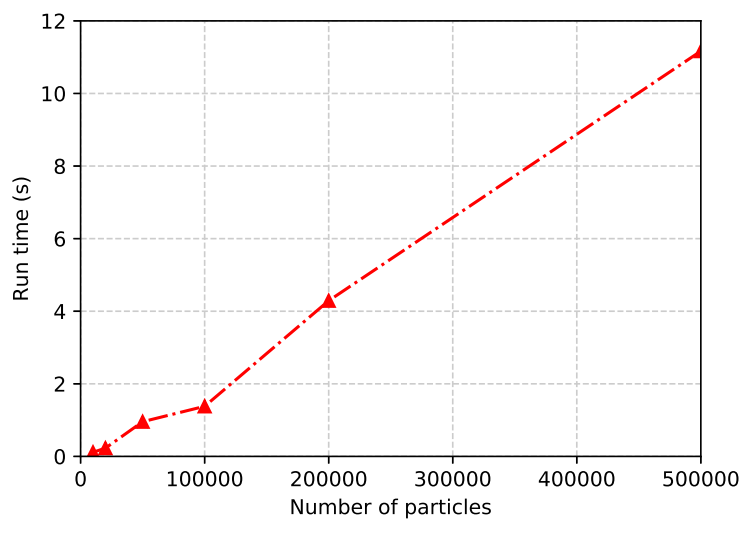

Figure 3: Performance of Fast Multipole Method (ExaFMM) on large collections of particles, with an expansion order $p=6$, and a maximum of 50 particles per cluster. 
pertaining to this study, meanwhile the reader is referred to the literature for an in-depth description of the FMM. ${ }^{30,31}$

Applying the FMM to the vortex particle method, the vector-potential field $\boldsymbol{\psi}$ in Eq. (14) can be efficiently evaluated for all particles at a computational effort $\mathcal{O}(N)$, which is a significant improvement compared to the direct calculation $\mathcal{O}\left(N^{2}\right)$. Figure 2 compares the performance of FMM versus the direct calculation for a small number of particles uniformly distributed, and Figure 3 shows this performance scaling linearly in evaluations on the order of the hundreds of thousands particles. For this study, the open-source code ExaFMM developed by Barba and Yokota ${ }^{16,32}$ has been modified and embedded into our code for the computation of the vorticity-induced velocity (Eq. (33)) and vortex stretching (Eq. (34)). ExaFMM is a 3D FMM modular code that is highly parallel and GPU-capable. The FMM calculation of vortex stretching is explained in the following section.

\section{II.A.5. Vortex Stretching Calculated Through Complex-step Derivative Approximation}

For the vortex particle method, we are interested in determining the vorticity-induced velocity $\mathbf{u}$ and vortex stretching $(\boldsymbol{\Gamma} \cdot \nabla) \mathbf{u}$ rather than the vector potential $\boldsymbol{\psi}$ calculated by the classic FMM, but these fields can be obtained from the derivatives of $\boldsymbol{\psi}$ as

$$
\begin{aligned}
& \mathbf{u}=\nabla \times \boldsymbol{\psi} \\
& (\boldsymbol{\Gamma} \cdot \nabla) \mathbf{u}=\left[\begin{array}{lll}
\frac{\partial^{2} \psi_{z}}{\partial x \partial y}-\frac{\partial^{2} \psi_{y}}{\partial x \partial z} & \frac{\partial^{2} \psi_{z}}{\partial y^{2}}-\frac{\partial^{2} \psi_{y}}{\partial y \partial z} & \frac{\partial^{2} \psi_{z}}{\partial z \partial y}-\frac{\partial^{2} \psi_{y}}{\partial z^{2}} \\
\frac{\partial^{2} \psi_{x}}{\partial x \partial z}-\frac{\partial^{2} \psi_{z}}{\partial x^{2}} & \frac{\partial^{2} \psi_{x}}{\partial y \partial z}-\frac{\partial^{2} \psi_{z}}{\partial y \partial x} & \frac{\partial^{2} \psi_{x}}{\partial z^{2}}-\frac{\partial^{2} \psi_{z}}{\partial z \partial x} \\
\frac{\partial^{2} \psi_{y}}{\partial x^{2}}-\frac{\partial^{2} \psi_{x}}{\partial x \partial y} & \frac{\partial^{2} \psi_{y}}{\partial y \partial x}-\frac{\partial^{2} \psi_{x}}{\partial y^{2}} & \frac{\partial^{2} \psi_{y}}{\partial z \partial x}-\frac{\partial^{2} \psi_{x}}{\partial z \partial y}
\end{array}\right]\left[\begin{array}{c}
\Gamma_{x} \\
\Gamma_{y} \\
\Gamma_{z}
\end{array}\right],
\end{aligned}
$$

hence, in implementing the FMM, it is necessary to include the calculation of the Jacobian and Hessian matrix of the vector potential, containing the first and second derivatives of $\boldsymbol{\psi}$, respectively.

The calculation of first and second derivatives of $\boldsymbol{\psi}$ is fairly simple in the case of direct $\mathcal{O}\left(N^{2}\right)$ calculation since it can be carried out algebraically; on the other hand, their computation through multipole and local expansions (Eqs. 31 and 32) requires an involved process. The difficulty arises when differentiating over the spherical harmonic functions while trying to maintain a computational complexity $\mathcal{O}(N)$ in the overall process. Other authors have taken a variety of different approaches for the calculation of derivates. For the first derivative, most authors derive and implement the analytical expression from local and multipole expansions without much problem (a detailed derivation can be found in Berdowski's dissertation ${ }^{1}$ ). However, in calculating the second derivative authors have differed between deriving an analytical expression, ${ }^{4,33}$ and computing the derivates through finite difference. ${ }^{1}$ The former approach is carried out with exact precision, with the drawback of making the FMM even more difficult to implement and maintain. On the other hand, the latter has the advantage of simplicity while forfeiting numerical accuracy. For the work presented in this paper, we introduce a unique approach: the calculation of vortex stretching through the complex-step derivative approximation. This approach is conceptually simple and easy to implement, while achieving machine precision.

The complex-step derivative approximation (CSDA) is a method for approximating "derivatives of real functions using complex variables which avoids the subtractive cancellation errors inherent in the classical derivative approximations". ${ }^{34}$ Originating from the work of Lyness and Moler ${ }^{35,36}$ five decades ago, CSDA was reintroduced by Squire and $\operatorname{Trapp}^{34}$ in 1998. Since then, this method has been applied for sensitivity analysis in aerospace and multidisciplinary design optimization. ${ }^{37-39}$ The method is derived from the Taylor expansion of a complex function as described below.

Let the real-variable analytic function $f: \mathbb{R} \rightarrow \mathbb{R}$ be generalized to its complex-variable form $f^{*}: \mathbb{C} \rightarrow \mathbb{C}$, and $x_{0} \in \mathbb{R}$ an arbitrary point where we desire to evaluate the first derivative of $f$. Let $h \in \mathbb{R}$ be an arbitrarily small step, the function $f^{*}$ can be Taylor expanded around $x_{0}+i h$ as

$$
f^{*}\left(x_{0}+i h\right)=f\left(x_{0}\right)+i h \frac{\mathrm{d} f}{\mathrm{~d} x}\left(x_{0}\right)-\frac{h^{2}}{2 !} \frac{\mathrm{d}^{2} f}{\mathrm{~d} x^{2}}\left(x_{0}\right)-\frac{i h^{3}}{3 !} \frac{\mathrm{d}^{3} f}{\mathrm{~d} x^{3}}\left(x_{0}\right)+\ldots
$$


Taking the imaginary value of $f^{*}\left(x_{0}+i h\right)$ and dividing by $h$

$$
\begin{aligned}
\frac{\operatorname{Im}\left(f^{*}\left(x_{0}+i h\right)\right)}{h} & =\frac{\mathrm{d} f}{\mathrm{~d} x}\left(x_{0}\right)-\frac{h^{2}}{3 !} \frac{\mathrm{d}^{3} f}{\mathrm{~d} x^{3}}\left(x_{0}\right)+\ldots \\
& =\frac{\mathrm{d} f}{\mathrm{~d} x}\left(x_{0}\right)-\mathcal{O}\left(h^{2}\right),
\end{aligned}
$$

the derivative of $f$ is then obtained as

$$
\frac{\mathrm{d} f}{\mathrm{~d} x}\left(x_{0}\right)=\frac{\operatorname{Im}\left(f^{*}\left(x_{0}+i h\right)\right)}{h}+O\left(h^{2}\right) .
$$

Let us now choose an $h$ sufficiently small — say $h=10^{-30}$ for double floating point precision -, and we have obtained a numerical approximation of the derivative of $f$ that is as accurate as machine precision.

CSDA can be directly applied to any real-variable real-valued function $f: \mathbb{R} \rightarrow \mathbb{R}$ by generalizing the function to the complex plane $f^{*}: \mathbb{C} \rightarrow \mathbb{C}$ and using this plane for carrying on the numerical differentiation. However, multipole and local expansions of the FMM (Eqs. 31 and 32) are computed through complexvalued functions $Y: \mathbb{R} \rightarrow \mathbb{C}$ already using the complex plane that would normally be used for the CSDA, requiring the formulation of CSDA in a multicomplex space. Hence, we will now define the multicomplex arithmetic needed for implementing CSDA into the FMM.

Let the complex set $\mathbb{C}^{1}$ with imaginary unit $i_{1}$ be $\mathbb{C}^{1}=\left\{x+i_{1} y / x, y \in \mathbb{R}\right\}$, and let us define the multicomplex set $\mathbb{C}^{2}$ with imaginary unit $i_{2}$ as $\mathbb{C}^{2}=\left\{z+i_{2} w / z, w \in \mathbb{C}^{1}\right\}$, any complex-valued analytical function $Y: \mathbb{R} \rightarrow \mathbb{C}$ will then be generalized into the multicomplex space $\mathbb{C}^{2}$ as $Y^{*}: \mathbb{C}^{1} \rightarrow \mathbb{C}^{2}$. In this generalization we have transformed the set $\mathbb{R}$ into $\mathbb{C}^{1}$, and $\mathbb{C}$ into $\mathbb{C}^{2}$; meaning that a variable $x$ originally in $\mathbb{R}$ now becomes $x^{*}=x+i_{1} 0$, and a variable $z=a+i b$ originally in $\mathbb{C}$ now becomes $z^{*}=a^{*}+i_{2} b^{*}=$ $\left(a+i_{1} 0\right)+i_{2}\left(b+i_{1} 0\right)=a+i_{2} b$. With this definition, the axis $i_{1}$ will carry the CSDA value and is not intended to interact with the axis $i_{2}$-hence, the product $i_{1} i_{2}=i_{2} i_{1}$ will purposefully remain undefined as to avoid any unintended arithmetic. The derivative will then be computed as

$$
\frac{\mathrm{d} Y}{\mathrm{~d} x}(x) \approx \frac{\operatorname{Im}_{1}\left(Y^{*}\left(x+i_{1} h\right)\right)}{h} .
$$

Since multipole and local expansion are computed through spherical harmonic functions $Y_{n}^{m}: \mathbb{R}^{2} \rightarrow \mathbb{C}$ of the form

$$
Y_{n}^{m}(\theta, \phi)=\sqrt{\frac{(n-|m|) !}{(n+|m|) !}} \cdot P_{n}^{|m|}(\cos \theta) e^{i m \phi},
$$

where $P_{n}^{|m|}$ are the associated Legendre functions, we now show how this multicomplex transformation applies to the complex exponential and complex product. Let the complex-valued function $Y: \mathbb{R} \rightarrow \mathbb{C}$ be defined as $Y(x)=e^{i x}$, the derivative is easily determined analytically as $\frac{\mathrm{d} Y}{\mathrm{~d} x}(x)=i e^{i x}$. On the other hand, its multicomplex generalization $Y^{*}: \mathbb{C}^{1} \rightarrow \mathbb{C}^{2}$ is defined as

$$
Y^{*}(z)=e^{i_{2} z}
$$

and the derivative $\frac{\mathrm{d} Y}{\mathrm{~d} x}(x)$ is computed through CSDA (Eq. (38)) as

$$
\begin{aligned}
\frac{\mathrm{d} Y}{\mathrm{~d} x}(x) \approx \frac{\operatorname{Im}_{1}\left(Y^{*}\left(x+i_{1} h\right)\right)}{h} & =\frac{1}{h} \operatorname{Im}_{1}\left(e^{i_{2}\left(x+i_{1} h\right)}\right) \\
& =\frac{1}{h} \operatorname{Im}_{1}\left(e^{i_{2} x} e^{i_{1}\left(i_{2} h\right)}\right) \\
& =\frac{1}{h} \operatorname{Im}_{1}\left(e^{i_{2} x}\left(\cos h+i_{1} \sin i_{2} h\right)\right) \\
& =\frac{1}{h}\left(e^{i_{2} x} \sin i_{2} h\right) \\
& =i_{2} e^{i_{2} x} \frac{\sinh h}{h} \quad, \text { where } \lim _{h \rightarrow 0} \frac{\sinh h}{h}=1 \\
& \stackrel{h \rightarrow 0}{=} i_{2} e^{i_{2} x},
\end{aligned}
$$




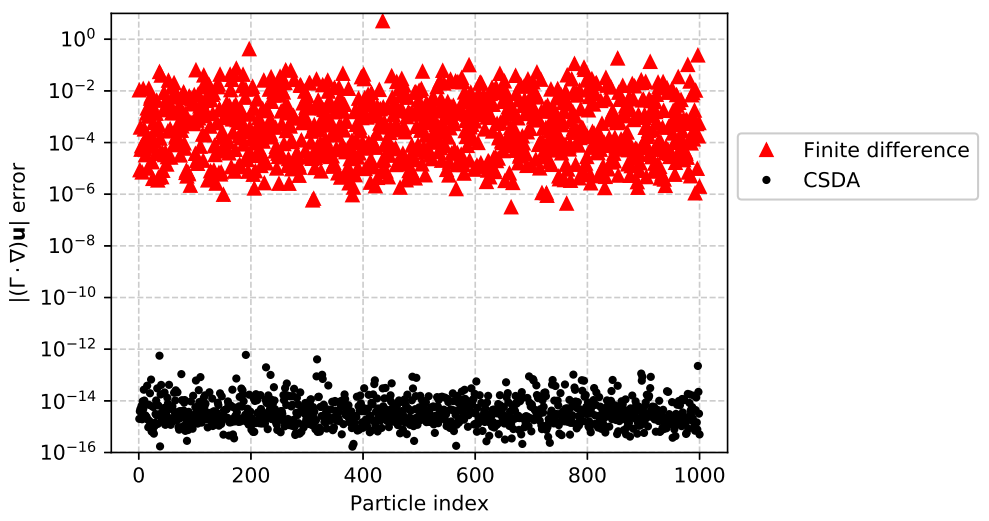

Figure 4: Error of vortex stretching at each particle computed through finite difference and CSDA, relative to the direct calculation.

obtaining the same result that the analytical derivation in the limit $h \rightarrow 0$.

Now we will show how CSDA is carried on the scalar-complex product. Let $Y: \mathbb{R} \rightarrow \mathbb{C}$ be defined as $Y(x)=c x=\left(c_{1}+i c_{2}\right) x$ with $c_{1}, c_{2} \in \mathbb{R}$, the derivative is trivially $\frac{\mathrm{d} Y}{\mathrm{~d} x}(x)=c$. Defining its multicomplex generalization $Y^{*}: \mathbb{C}^{1} \rightarrow \mathbb{C}^{2}$ as

$$
Y^{*}(z)=c^{*} z
$$

with $c^{*} \in \mathbb{C}^{2} / c^{*}=c_{1}^{*}+i_{2} c_{2}^{*}=\left(c_{1}+i_{1} 0\right)+i_{2}\left(c_{2}+i_{1} 0\right)$, the derivative $\frac{\mathrm{d} Y}{\mathrm{~d} x}(x)$ is then computed through CSDA as

$$
\begin{aligned}
\frac{\mathrm{d} Y}{\mathrm{~d} x}(x) \approx \frac{\operatorname{Im}_{1}\left(Y^{*}\left(x+i_{1} h\right)\right)}{h} & =\frac{1}{h} \operatorname{Im}_{1}\left(c^{*}\left(x+i_{1} h\right)\right) \\
& =\frac{1}{h} \operatorname{Im}_{1}\left(\left(c_{1}+i_{2} c_{2}\right)\left(x+i_{1} h\right)\right) \\
& =\frac{1}{h}\left(c_{1}+i_{2} c_{2}\right) h \\
& =c_{1}+i_{2} c_{2} \\
& =c^{*} .
\end{aligned}
$$

The complex exponential and the complex product are not only good example cases of multicomplex arithmetic, but they are also the only two special operations needed for carrying on the CSDA through the multipole and local expansions of the FMM.

In order to compare accuracy between finite difference and CSDA, vortex stretching in 1000 randomlygenerated particles was computed through direct calculation, FMM with finite difference, and FMM with CSDA. The error relative to the direct calculation is shown in Figure 4 for each particle, evidencing the subtractive and round off error introduced by the finite difference, meanwhile CSDA approaches floatingpoint precision.

\section{II.A.6. Other Schemes}

In the current state of our VPM code, vortex stretching is solved in the transposed scheme as explained in Winckelmann's thesis, ${ }^{40}$ and the divergence of the vorticity field is relaxed through the relaxation scheme developed by Pedrizzeti. ${ }^{41}$ The time integration of the governing equations is done implementing a lowstorage third-order Runga-Kutta scheme. ${ }^{42}$ No provisions against Lagrangian grid distortion — other than the initial particle core overlap - are implemented. As we will be shown in Section IV, this will lead us to exclude cases that are prone to numerical instability from this study. In future work, a particle splitting/mixing scheme will be implemented to address Lagrangian distortion. 


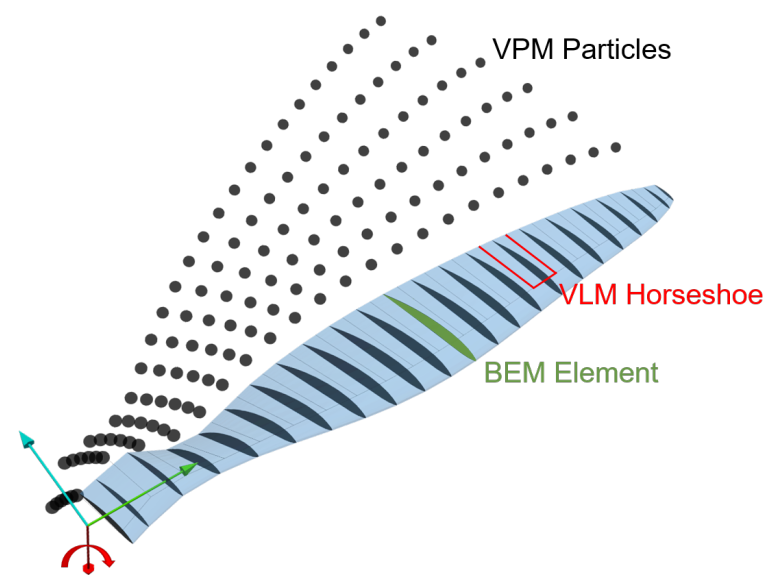

Figure 5: Individual blade of the APC 10x7 propeller model.

\section{II.B. Propeller Model}

In this study, each blade of the propeller is modeled as a rotary lifting surface where the physics of interest have been broken down into three aspects: load distribution, blade-induced velocity, and wake-induced velocity. The load distribution is calculated through blade element momentum (BEM) theory and used for deriving the circulation along a vortex lattice representing the lifting surface. In turn, the vortex lattice is used as a framework for manipulating the rotating geometry, calculating the direction and strength of vortex particles being shed off the trailing edge, and calculating the velocity that the lifting surface induces on nearby particles. Wake-induced velocity and unsteady wake dynamics are obtained from the vortex particle code. All simulations will be based on the geometry and performance of the APC thin-electric 10x7 propeller. This propeller is a hobby-grade model commonly used on small unmanned aircraft, it is readily available, and its 10-inch diameter makes it a good fit for mid-size wind tunnel testing. Moreover, its performance has been measured and indexed in the UIUC Propeller Database, ${ }^{43}$ and verified by McCrink and Gregory ${ }^{44}$ both experimentally and through BEM. Figure 5 shows the APC 10x7 blade geometry discretized into 21 blade elements and vortex lattice along with the particles shed in the first 6 time steps of simulation. All codes used in this propeller model are in-house developed. The following paragraphs detail the implementation and coupling of blade element momentum and vortex lattice codes with the VPM.

In the initial step, aerodynamic characteristics of each BEM element are calculated and used for solving the lift distribution. At each cross section of the blade, two-dimensional aerodynamic airfoil characteristics are computed through XFOIL at the respective local Reynolds number, and a Prandtl-Glauert compressibility correction is applied to the lift curve. Both force curves are adjusted to capture 3D drag and stall-delay effects $^{45}$ encountered in rotary wings, and the Viterna method ${ }^{46}$ is applied to obtain post-stall $\pm 180^{\circ}$ extrapolations of these curves. A formulation with guaranteed convergence ${ }^{47}$ is then applied to the BEM problem, and near and far field induced velocities are solved to finally derive the load and circulation distribution. This method is implemented and made available through the open-source code CCBlade ${ }^{\mathrm{a}}$. All simulations in this study will be run at a diameter-based Reynolds number of $1.5 \times 10^{6}$ relative to the local rotational velocity of the blade at $70 \%$ of the blade span. The advance ratio will be varied by increasing the freestream velocity while maintaining the rotation of the propeller at 9400 RPM (resulting in a base tip Mach number of 0.36 ). The performance of the APC 10x7 predicted by BEM under such conditions is compared with experimental measurements in Figure 6 showing close agreement.

Regarding the blade-wake coupling, we have observed that omitting the velocity that is induced by the lifting surface leads to the development of an inaccurate wake geometry. The passage of the blade nearby the preceding vortex tip has the effect of impelling the vortex core further downstream; hence, disregarding such induced velocity leads to a helicoid wake that is axially underexpanded in the near field. This is especially true for hover or low-advance-ratio simulations where self-induced velocity is the predominant mechanism of wake convection. The blade-induced velocity is introduced into the VPM's governing equations (Eqs. 7 and 8) as a scalar-potential velocity field $\mathbf{u}_{\phi}$, where $\mathbf{u}$ becomes $\mathbf{u}=\mathbf{u}_{\infty}+\mathbf{u}_{\boldsymbol{\omega}}+\mathbf{u}_{\phi}$, with $\mathbf{u}_{\infty}$ is the

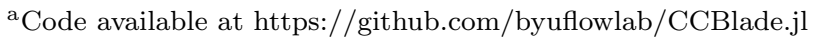



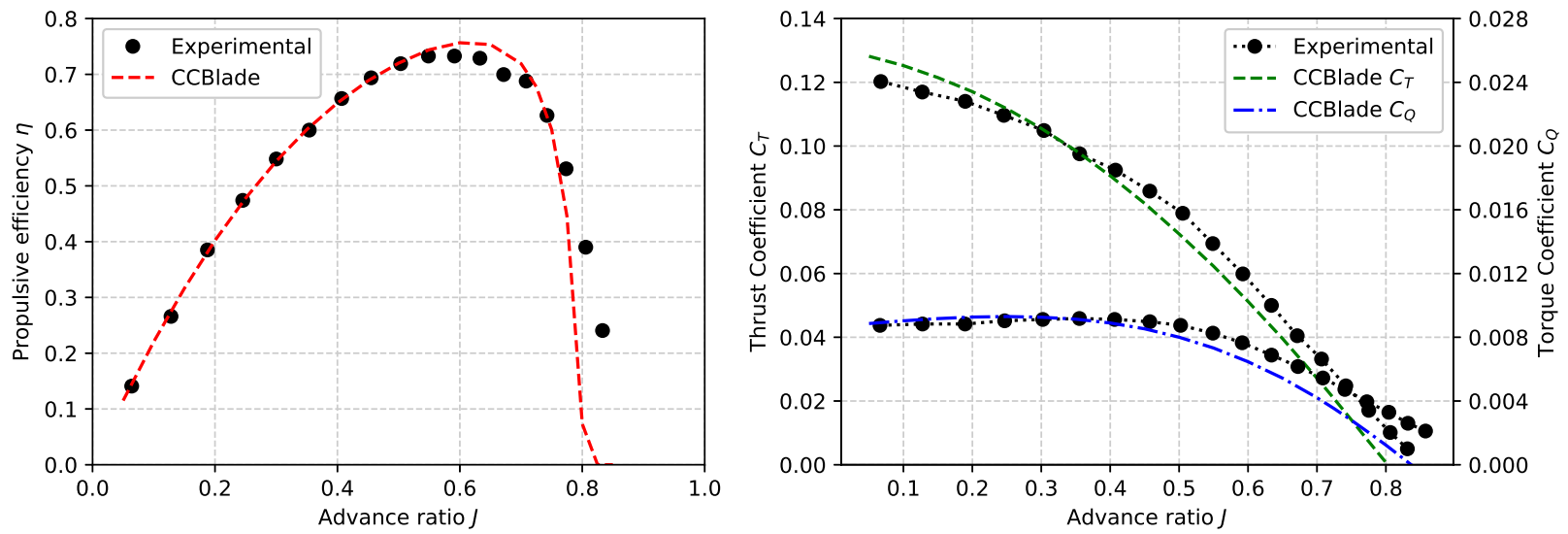

Figure 6: CCBlade's predicted performance of APC 10x7 propeller compared to experimental measurements reported by McCrink and Gregory. ${ }^{44}$

freestream velocity, $\mathbf{u}_{\boldsymbol{\omega}}$ is the velocity induced by the particle field (wake), and $\mathbf{u}_{\phi}$ is the velocity induced by the lifting surface. The governing equations not only require the evaluation of the velocity field $\mathbf{u}_{\phi}$, but vortex stretching (Eq. (8)) also requires the calculation of its derivatives.

In order to model the lifting surface, the circulation distribution derived from BEM is then used as the strengths of a vortex lattice with one chordwise horseshoe element. The Vortex Lattice Method (VLM) has been extensively developed in the literature. ${ }^{48}$ For the purpose of this propeller model, the VLM's semi-infinite vortex lines have been removed resulting in horseshoe elements made of one bound-vortex section at the quarter chord and two bound-vortex sections that extend from the quarter chord to the trailing edge as shown in Figure 5. At each trailing edge station a vortex particle is shed in the direction of freestream and local rotational velocity, with a strength proportional to the difference between contiguous VLM horseshoes. Thus the unsteady propeller wake is formed from VPM particles as the blades rotate. A general-geometry formulation of the VLM has been developed and made available through the open-source code FLOWVLM ${ }^{\mathrm{b}}$. The code allows the calculation of automatic gradients for optimization, which in this study is used for calculating the contribution of the lifting surface on VPM vortex stretching (Eq. 8).

The focus of this study is the modeling of unsteady wake dynamics and mixing in a multirotor configuration. Prop-on-prop interaction and an unsteady wake produce a load distribution along each blade that varies in time and azimuthal position, that in turn affects the strength of shed vortices. In an attempt to simplify computation, it will be assumed that this unsteady loading will have a second-order effect in the resulting wake geometry and dynamics, and will be neglected. Hence, in all simulations hereby reported the load distribution calculated through BEM will be kept constant as the wake develops, ignoring the effects of wake dynamics and prop-on-prop interaction on propeller performance. In future work, the effects of these interactions on propeller performance will be studied by closing the feedback loop between wake and load distribution.

\section{Preliminary Validation}

Since the work of Winckelmans, ${ }^{40}$ vortex rings have become the benchmark for vortex particle codes, for-in the words of Saffman - "one particular motion exemplifies the whole range of problems of vortex motion and is also a commonly known phenomenon, namely the vortex ring ... Their formation is a problem of vortex sheet dynamics, the steady state is a problem of existence, their duration is a problem of stability, and if there are several we have a problem of vortex interactions". ${ }^{49}$ A vortex ring is a toroidal vortex tube that impels itself axially by virtue of the flow field induced around its circulation. As suggested in the work of Berdowski, ${ }^{1}$ the dynamics of vortex rings embody the fundamental phenomena observed in a propeller wake. The wake originated at the plane of rotation impels itself downstream with self-induced velocity analogous to a vortex ring. As the wake travels downstream, tip vortices and inner vortex sheets build a regular helical geometry that eventually transitions to a turbulent vortex breakdown. Experimental studies

\footnotetext{
${ }^{\mathrm{b}}$ Code available at https://github.com/byuflowlab/FLOWVLM
} 
have observed that the transition to vortex breakdown is associated with filament instabilities leading to stretching, leapfrogging, and pairing of tip vortices, analogous to the leapfrogging of coaxial vortex rings. ${ }^{50}$ Hence, in this section we will use the vortex particle code to model some of the dynamics of vortex rings, and compare its predictions to either analytical solutions or experimental results.

\section{III.A. Self-induced Velocity and Viscous Diffusion}

In the actuator disk model, the wake shed downstream of the disk is composed of a series of vortex rings that produce the induced velocities observed at the plane of rotation and far field. Even though the propeller model developed in this study (Section II.B) implements a rotary wing shedding vortex particles in a helical manner rather than an actuator disk, the self-propelled vortex ring provides a preliminary validation of the physics behind the velocity induced by the wake. Neglecting viscous effects, the self-induced velocity of a vortex ring's centroid is calculated analytically ${ }^{51}$ as

$$
U=\frac{\Gamma}{4 \pi R}\left(\ln \left(\frac{8 R}{a}\right)-\frac{1}{2}\right)
$$

with $\Gamma$ the ring's circulation, $R$ the radius of the ring, and $a$ the core's radius. Following the discretization used by Winckelmans, ${ }^{40}$ a toroidal vortex ring of $\Gamma=1 \mathrm{~m}^{2} / \mathrm{s}, R=1 \mathrm{~m}$, and $a / R=0.15$ was simulated under both inviscid and viscous conditions. Both rings were discretized into 200 cross section and the particlesmoothing radius $\sigma$ was made equal to the ring's core $a$. The inviscid ring was modeled with three concentric layers of particles along the toroid's centerline, adding a total of 5000 particles. The simulation resulted in a centroid velocity of $0.2769 \mathrm{~m} / \mathrm{s}$, meanwhile the analytical solution predicted a velocity of $0.2767 \mathrm{~m} / \mathrm{s}$, resulting in an error of only $0.09 \%$. This accuracy is a favorable indicator for the calculation of wake-induced velocity when the VPM is applied to the propeller case.

The viscous ring was modeled as a single line of particles positioned at the toroid's centerline initially containing the entire vortex strength. Ten layers of particles with zero vortex strength were placed around the centerline to allow for the Particle Strength Exchange scheme to diffuse the vortex core outwards, adding a total of 88200 particles. The simulation was run at a Reynolds number $\operatorname{Re}=\Gamma / \nu$ of 400 . Figure 7 shows a cross sectional view of the vortex ring as the simulation progresses, where the vortex core is fully contained at the centerline at $t=0 \mathrm{~s}$, and rapidly diffuses out in the first few seconds. By $t=4.5 \mathrm{~s}$ the diffusion front has already reached the outmost layer of particles, and without any more particles to diffuse the core further out, the viscous diffusion scheme spreads the vectorial circulation until it is a uniform field across the particles. After 18 seconds it is seen that the vortex core has completely diffused through the 10 layers of particles, with all the particles having reached the same vortex strength. In Figure 8 the position of the ring's centroid is plotted as a function of time, and is compared to both the inviscid simulation and the

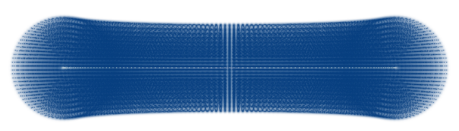

(a) $t=0 \mathrm{~s}$

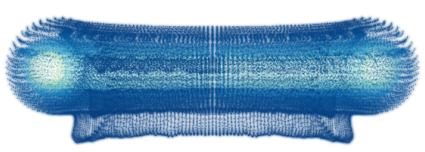

(d) $t=1.8 \mathrm{~s}$

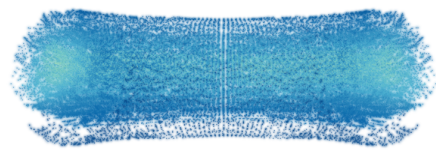

(g) $t=9.0 \mathrm{~s}$

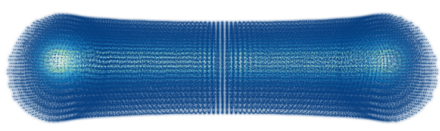

(b) $t=0.5 \mathrm{~s}$

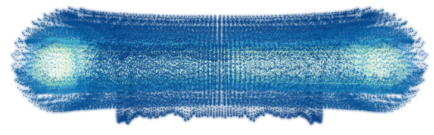

(e) $t=2.7 \mathrm{~s}$

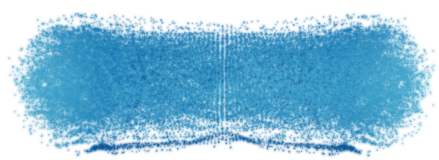

(h) $t=18.1 \mathrm{~s}$

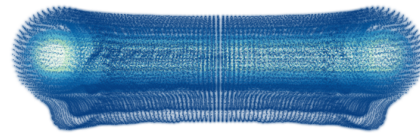

(c) $t=1.4 \mathrm{~s}$

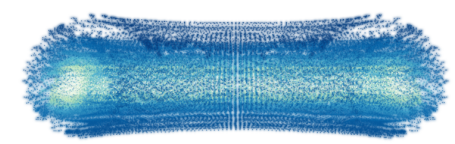

(f) $t=4.5 \mathrm{~s}$

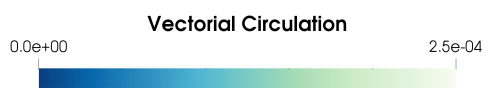

Figure 7: Evolution of the viscous vortex ring at $\mathrm{Re}=400$ where it can be seen the vortex core diffusing outward as time progresses. 


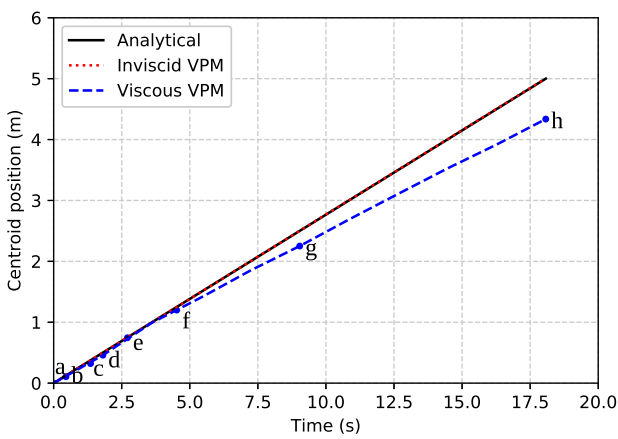

Figure 8: Comparison of self-induced velocities between the viscous ring and the inviscid case. Labels correspond to frames shown in Figure 7.

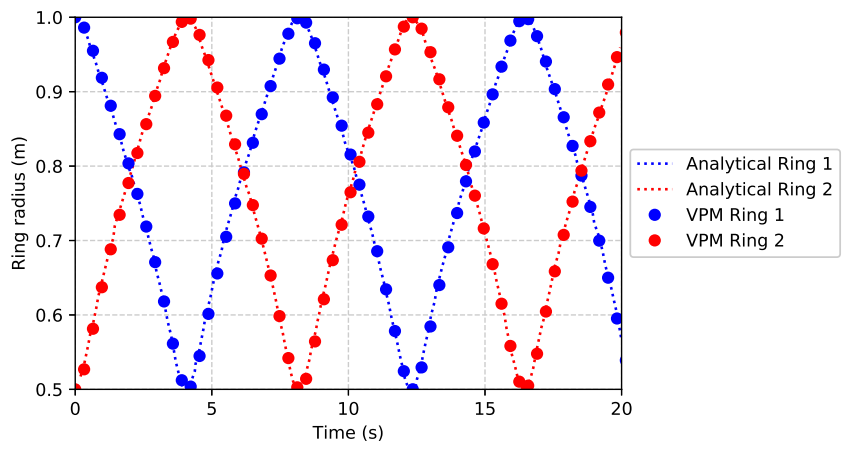

Figure 9: Comparison of analytically-predicted versus VPM-predicted leapfrog dynamics. Analytical solution extracted from Berdowski's thesis. ${ }^{1}$

inviscid analytical solution (Eq. (54)). There it can be seen that the viscous ring has the same velocity as the inviscid case while the diffusion front is travelling outward $(0<t<4.5 \mathrm{~s})$, but it immediately deviates once further viscous diffusion is obstructed due to the limited number of particles. The diffusion of the vortex core as observed verifies the correct implementation of the Particle Strength Exchange scheme.

\section{III.B. Vortex Stretching}

Another case of interest is the ring interaction known as leapfrogging, where two coaxial rings successively move past each other as they travel. The rings successively stretch and contract as to give way to the passing ring, hence this case will serve for testing the stability of the VPM during vortex stretching. Since the vortex particle method works as a discretization of vortex filaments, the method must ensure connectivity between particles on the same filament before vortex breakdown; hence, the leapfrog case will also serve as a test of conservation of connectivity as the rings stretch and contract. Two concentric vortex rings with circulations $\Gamma_{1}=\Gamma_{2}=1 \mathrm{~m}^{2} / \mathrm{s}$, initial radii $R_{1}=1.0 \mathrm{~m}, R_{2}=0.5 \mathrm{~m}$, and cores $\sigma_{1}=\sigma_{2}=0.1$ were simulated as to initiate the leapfroging dynamics, initially with Ring 2 inside Ring 1 . Figure 9 compares the successive expansion and contraction of the rings as predicted through the VPM to results obtained from the analytical solution of this phenomena found in the work of Konstantinov ${ }^{52}$ and reported by Berdowski, ${ }^{1}$ showing exact agreement.

\section{III.C. Vortex Collision and Reconnection}

Since the work of Winckelmans almost 30 year ago, the "knot" problem has become a benchmarking case for vortex particle codes. The problem defines two rings that are initially linked (or knotted), introducing a non-zero helicity. As the simulation progresses, the rings collide breaking up their original connection and reconnecting into a new pair of rings. This case contains some of the features of wake interaction where vortex tubes from different propellers mix forming a new coherent body; it also tests the numerical stability of the VPM as the rings go through extreme stretching requiring loss of connectivity and reconnection. Even though the knot problem has been extensively studied, it was thought to be only a computational problem, and impossible to be reproduced in the laboratory. Only five years ago, the long-sought-after knotted problem was successfully reproduced experimentally by Kleckner and Irvine ${ }^{53}$ by an ingenious design of linked circular wings. To the best of our knowledge, this is the first time the knotted problem is compared between VPM and actual experimental dynamics.

Following the experimental configuration described by Kleckner and Irvine, ${ }^{53}$ two linked rings of radii $R=26.7 \mathrm{~mm}$ and circulation $\Gamma=3.1 \times 10^{-2} \mathrm{~m}^{2} / \mathrm{s}$ at a mutual inclination of $20^{\circ}$ were simulated at a Reynolds number of $\operatorname{Re}=\Gamma / \nu=3.1 \times 10^{4}$ and core radii of $\sigma / R=0.07$. The evolution of the rings is compared to the dynamics observed by Kleckner and Irvine ${ }^{53}$ in Figure 10. As the rings initially travel, their inner sections bend and stretch, and by $t=111 \mathrm{~ms}$ they come into contact simultaneously at two points. The filaments briefly lose connection at these points, reconnect respective inner and outer sections of the rings, and create a new pair of unlinked rings. The new inner-sections ring presents a topology that has been highly stretched and deformed (see frames at $t=134 \mathrm{~ms}$ ). Simulations showed that the ring is very unstable and bursts 


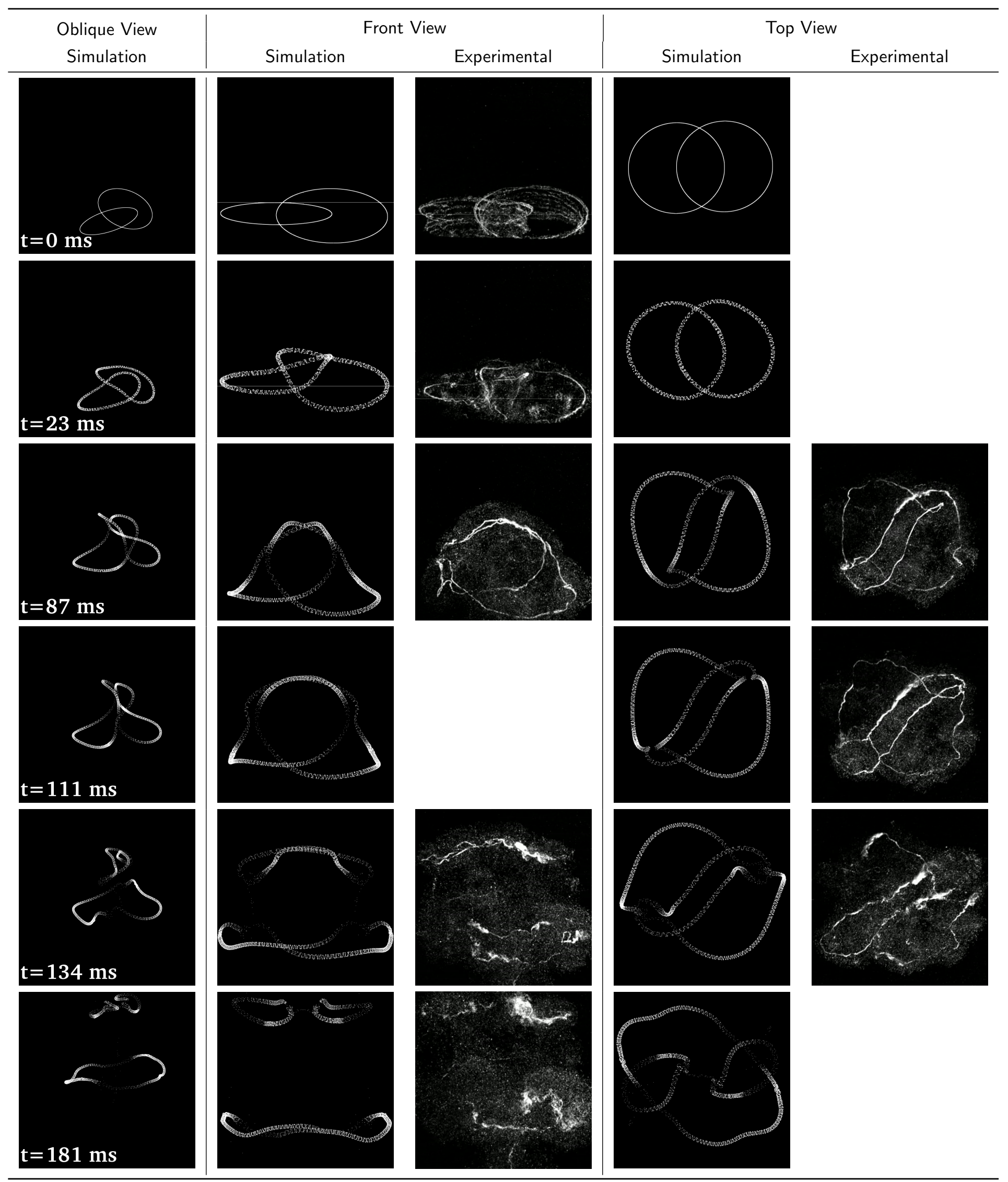

Figure 10: Comparison of simulated and experimental evolution of the knotted rings problem. Experimental frames reproduced by permission from Springer Nature: Springer Nature Physics. D. Kleckner and W. T. M Irvine, Creation and dynamics of knotted vortices (2013). doi: 10.1038/NPHYS2560. 
into vortex breakdown soon after reconnection. However, under a narrow range of relaxation, stability was extended and it was observed that the ring collides with itself forming a third ring shortly before breakdown as shown in frames at $t=181 \mathrm{~ms}$. Whether this is physically true or a product of numerical relaxation is unclear, but it seems to be hinted by the experimental observation. The experiment reports that the rings first reconnect after $208 \mathrm{~ms}$, meanwhile the simulation predicted the event after $134 \mathrm{~ms}$. The difference in time scales could be due to using a different vortex core size than the experimental ring; in spite of this, the succession of events and reconnection dynamics show good qualitative agreement, verifying the capacity of VPM to model mixing of vortical bodies.

\section{Results and Discussion}

In the absence of a mid/high fidelity model fit for conceptual design, wake interactions encountered in distributed propulsion have often been modeled with low-fidelity analytical tools borrowed from conventional aircraft design. However, many of these tools introduce assumptions that may not be applicable during the complex interactions between rotors and lifting surfaces. It is our purpose to compare one of these tools - momentum theory - with the unsteady dynamics predicted by VPM. First, a qualitative validation of unsteady wake dynamics predicted through VPM will be presented in Section IV.A, comparing the wake topology resulting from simulations to experimental results reported in the literature. In Section IV.B we will compare the theoretical near/far-field induced velocities to VPM in the stable wake region. In Section IV.C we will then compare the theoretical far-field induced velocity with what is predicted through VPM in the unstable vortex-breakdown region. Finally, in Section IV.D we will compare near and far field values of isolated propellers to the multirotor case where the wakes have been mixed.

\section{IV.A. Wake Topology}

In order to verify the modeling of unsteady dynamics, first we will look at the wake geometry predicted by the VPM. Even though the unsteady dynamics in a propeller wake can be expected to be deterministic and periodic in its early stage of development, experiments have shown multiple types of aperiodic behaviors as the wake convects further downstream even under controlled laboratory conditions. One source of these aperiodic behaviors is the inherent instability modes of helicoid vortex filaments. ${ }^{54} \mathrm{~A}$ stability analysis shows that wake stability is greatly dependent on the pitch of the helicoid as determined by advance ratio [55, Sec. 10.8.1], and experiments have also shown dependency on disk loading. ${ }^{50}$ Figure 11 shows the transition from the stable periodic region to an unstable vortex breakdown in the wake of a marine propeller visualized by cavitation bubbles. In this case, the mechanism of transition is the rise of small perturbations that develop into aperiodic leapfrogging dynamics and pairing of vortex filaments.

When modeling wake instability through VPM, attention must be paid that the onset of instability is due to a physical mechanism (e.g., vortex stretching, pairing, diffusion, etc), and not numerical error. We observed that the initial development of the wake plays a significant role in the resulting wake topology. The wake is convected downstream the plane of rotation due to two components: freestream, and self-induced velocity. This means that, in the absence of freestream, the wake is convected only due to self-induced

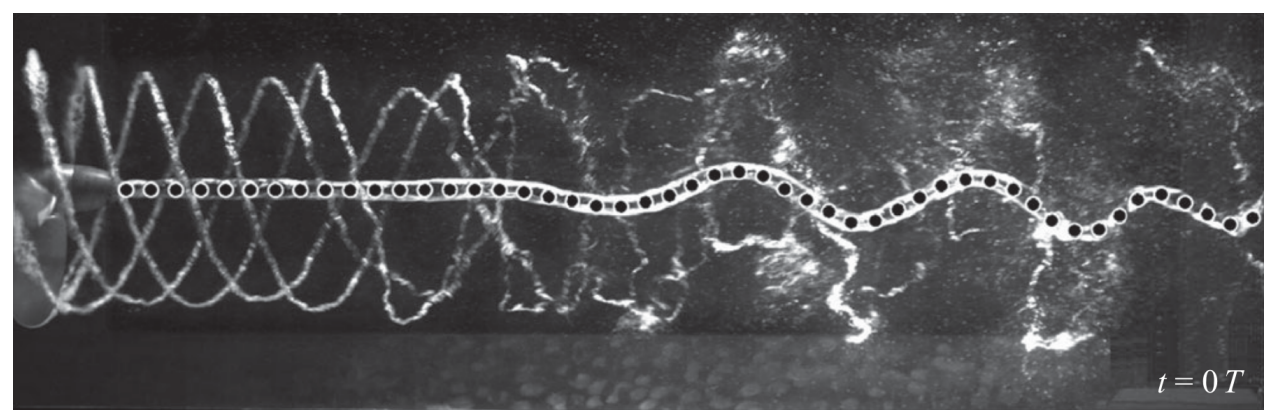

Figure 11: Marine propeller wake visualized through cavitation bubbles showing the transition from stable to unstable regions through leapfrogging. Reproduced by permission of Cambridge University Press from Felli et al..$^{50}$ 

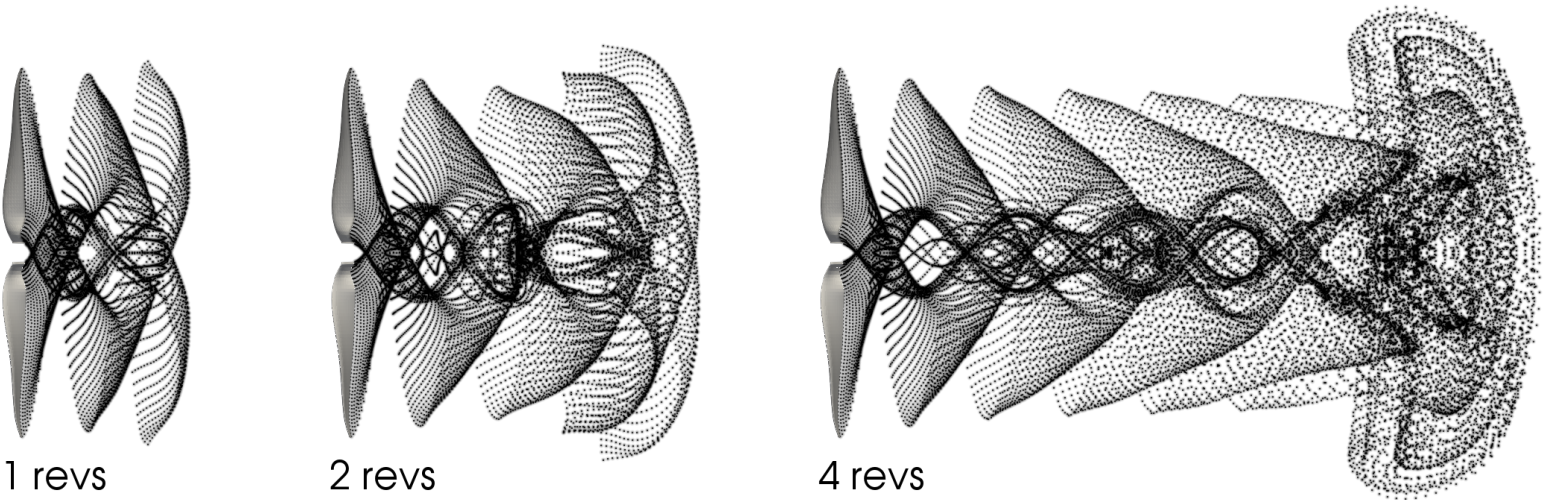

Figure 12: Formation of plume at wake front due to roll out of the inner vortex sheet.

velocity. ${ }^{\mathrm{c}}$ Hence, in the initial stage, the vortex particles tend to stay at the plane of rotation until a vortexring-like structure is formed and starts moving with a self-induced velocity. This creates a vortex front that forms the vortextube as it travels downstream. In turn, the vortextube fore of the vortex front pushes the inner vortex sheet ahead of the tip vortices, forcing it to roll out of the vortextube, thus creating a plume at the front of the vortextube. This is shown in Figure 12 displaying the first four revolutions of the APC 10x7 propeller at an advance ratio, $J$, of 0.35 . We observed that the formation of the plume involves an early vortex breakdown that eventually leads to numerical blow up if a large regularizing (smoothing) radii is not provided. Furthermore, static (no freestream) simulations showed that the plume also results in a premature onset of leapfrogging in its proximity, which in turn leads to a helicoid that is axially under-expanded and aperiodic in what should be the stable region. Lee and $\mathrm{Na}^{56}$ suggested that some of these dynamics can be avoided by slowly increasing the rotational speed of the propeller, but simulations showed that this only solved the issue of hub reversed flow encountered at low advance ratios, with the plume still being formed. In an attempt to deal with the plume, simulations were initiated with a wake in the shape of Landgrebe's rigid wake model ${ }^{57}$ along ten diameter lengths downstream, just to still observe the formation of the plume at the very end. Even locating it ten diameters downstream, the plume still onsets leapfrogging dynamics that are preceded by an axial compression of the wake in a spring-like reaction that is transmitted all the way back to the propeller plane.

The initiation plume was successfully removed with the following procedure. First, an arbitrary maximum shedding distance is defined such that any particle that passes this limit is removed from the field. Second, wake shedding is initiated, and the wake travels downstream as the plume is formed. Due to the deletion of particles at this plane, the plume is gradually removed as it crosses the limiting shedding distance. The leapfrogging dynamics initiated by the plume are also removed as they cross the limiting shedding distance. After the plume and its effect are removed, the wake attains a periodic, very stable behavior. Third, once stable dynamics are observed, the limiting shedding distance is moved further downstream, allowing a new plume to form, travel downstream, and be removed once again. Then the process is iterated. This procedure allowed us to reliably remove numerical instabilities introduced by the plume in simulations with an advance ratio higher than 0.3; however, we observed that simulations of lower advance ratios $(J<0.2)$ are easily prone to numerical blow up. Furthermore, no particle splitting/mixing scheme has currently been implemented in our the VPM code, which increases the difficulty of handling simulations that are numerically unstable. Hence, in the following sections we will limit our analyses to moderate/high advance ratios.

Figure 13 shows an example of the wake developed at $J=0.35$ were the topology is clearly identified. In the first three diameters downstream, the stable region is formed with periodic behavior. This is the region where the assumptions of momentum theory are valid, with the tip vortices in the near field going through a rapid radial contraction as they transition to the far field. Near and far field induced velocities calculated through VPM will be compared to the analytic results of momentum theory in Section IV.B. Short-wave instabilities start to develop about four diameters downstream, followed by the leapfrogging and pairing of tip-vortex filaments. All of these instabilities build up to about eight diameters downstream where vortex breakdown is made evident by the loss of all structure. It will be shown in Section IV.C that the theoretical

\footnotetext{
${ }^{\mathrm{c}}$ In addition to wake self-induced velocity, the lifting surface also contributes to the convection of the wake, but experience shows that this is only relevant during the first passage of the blade.
} 


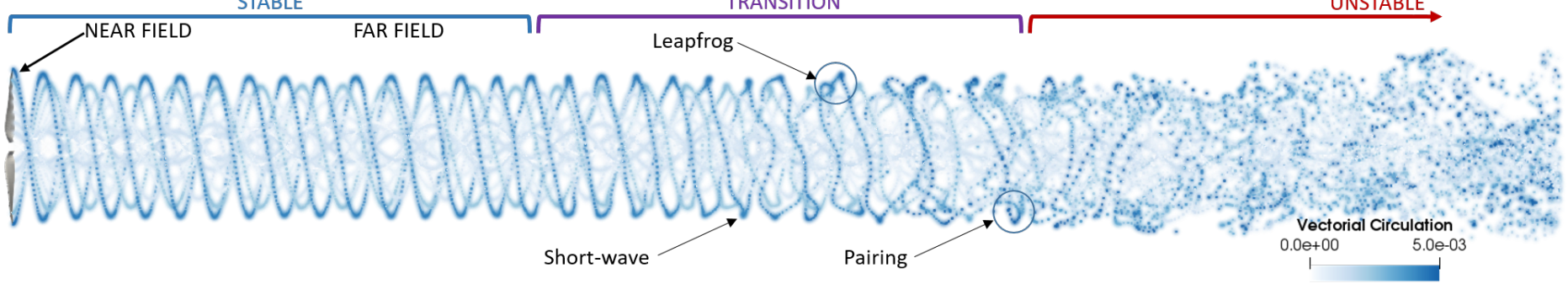

Figure 13: Wake of APC 10x7 as it develops ten diameters downstream showing both stable and unstable regions, and their transition through leapfrogging.

far-field values of induced velocities differ largely from their actual value in the unstable region. These dynamics show qualitative agreement with what is observed experimentally by Felli et al. ${ }^{50}$ (compare with Figure 11), hinting at the usefulness of VPM as a tool for thoroughly modeling unsteady wake dynamics.

\section{IV.B. Near to Far Field Transition}

In momentum theory, the propeller is modeled as an infinitely thin disk introducing a discontinuity in pressure. Assuming a rigid wake starting at the plane of rotation, it is found that induced velocities far downstream are exactly twice than at the disk. The induced axial velocity smoothly increases from zero upstream of the disk to its far-field value across the disk. The swirl induced velocity, in the other hand, faces an abrupt jump across the disk, being zero upstream of the disk, nonzero at the disk, and twice the disk value everywhere downstream. Even though the swirl induced by the rigid wake is a smooth monotonic function, the bound circulation representing the lifting surface at the disk introduces a singularity, inducing a velocity which cancels the wake-induced swirl everywhere upstream [58, Chapter 3]. This gives the analytical result that - under actuator disk and rigid wake assumptions - the far-field values of both axial and swirl induced velocities downstream the propeller are twice the near-field (disk) values, which become the near and far field assumptions of momentum theory.

The induced velocities were probed and averaged across sections of the VPM wake at $J=0.35$ shown in Figure 13. The velocities were also time-averaged to capture unsteady dynamics. Figure 14 compares the resulting average velocities to the near and far field of momentum theory. There it can be seen that the resulting swirl is zero in front of the plane of rotation $(x / D=-0.1)$, and abruptly jumps across the plane from the near field value $(x / D=0)$ to the far field value almost immediately downstream $(x / D=0.1)$. In the other hand, the axial velocity smoothly increases across the plane of rotation from the near field value at $x / D=0$, reaching the far field value one diameter downstream $(x / D=1.0)$. It is noteworthy to mention that all the time-averaged curves showed neglegible standard deviations (less than 1\%) verifying that the this region indeed presents a regular and periodic behavior. The swirl velocity shows good agreement with the near/far field values of momentum theory, meanwhile the axial velocity seems to shift inboard. With this we have verified the consistency between VPM and momentum theory; furthermore, we have seen the VPM's ability to characterize the near to far field transition in the stable region, which cannot be captured by momentum theory.

\section{IV.C. Stable to Unstable Region Transition}

Since momentum theory is based on the assumption of a rigid vortextube, we here explore its limitations in transition and vortex-breakdown regions. Figure 15 shows the velocities probed one diameter into the stable region $(x / D=1)$, six diameters downstream where leapfrogging is observed in the transition region $(x / D=6)$, and nine diameters downstream in the unstable region after vortex breakdown $(x / D=9)$. Shaded areas represent one standard deviation at every position of each curve during 20 propeller revolutions. In the transition region, both velocities tend to shift slightly inboard, and variation starts to grow at the center of the vortextube. In spite of this, the far-field value from momemtum theory still agrees fairly well with the transition average velocity. However, once the wake fully transitions to the unstable region, both curves seem to flatten out, widely deviating from the far field velocity of momentum theory, and a variability as large as 50\% from the average value is observed due to the turbulent behavior after vortex breakdown. These 

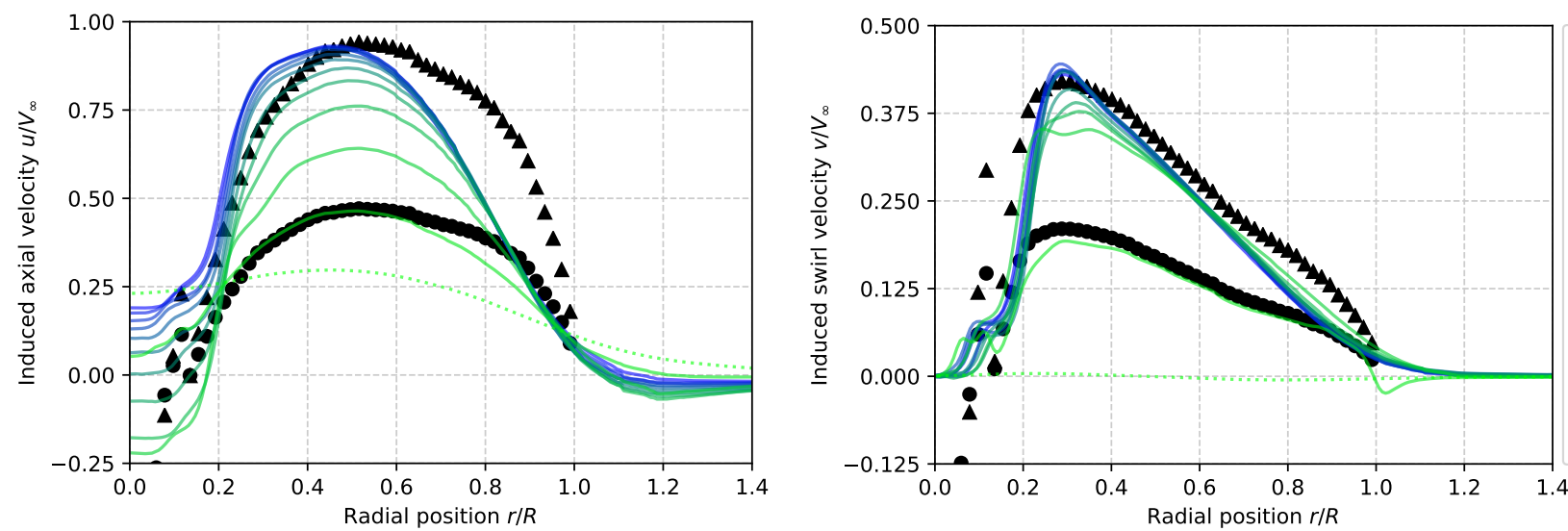

A Far field

- Near field

$x / D=1.0$

$x / D=0.9$

$x / D=0.8$

$x / D=0.7$

$x / D=0.6$

$x / D=0.5$

$x / D=0.4$

$x / D=0.3$

$x / D=0.2$

$x / D=0.1$

$\mathrm{x} / \mathrm{D}=0.0$ $x / D=-0.1$

Figure 14: Comparison between momentum theory's near/far-field velocities and VPM velocities across stable wake region, showing the ability of VPM to characterize the near to far field transition in the stable region.
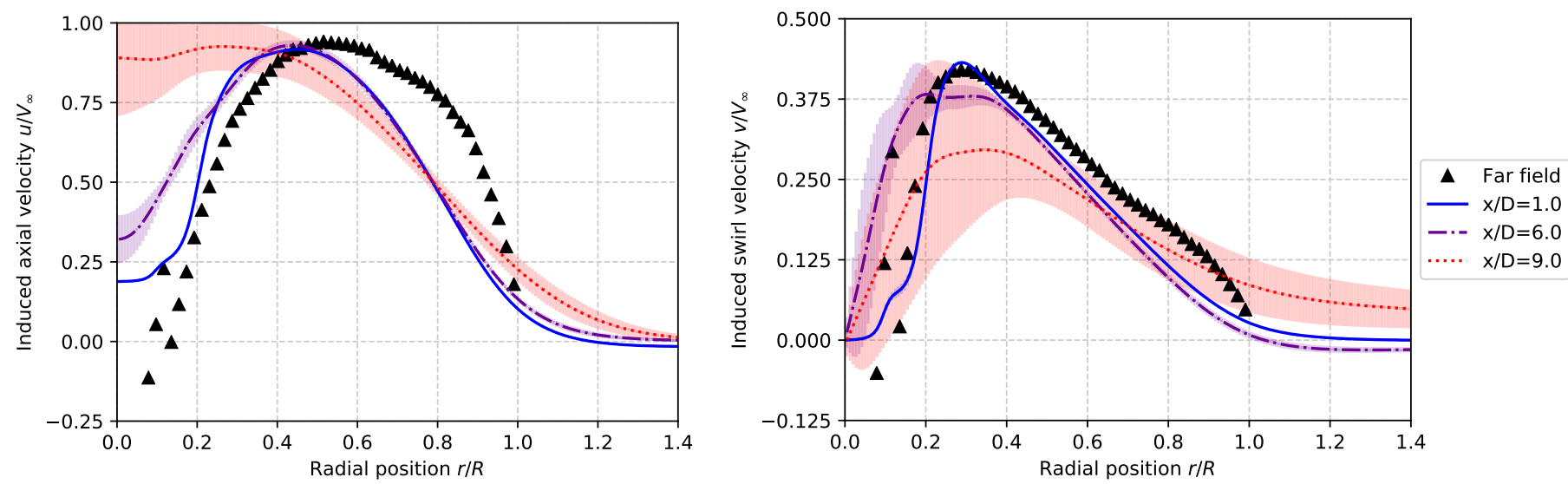

Figure 15: Comparison between momentum-theory far field induced velocities and time-average velocities across the unstable wake region obtained from VPM. Shaded areas represent one standard deviation at each position.
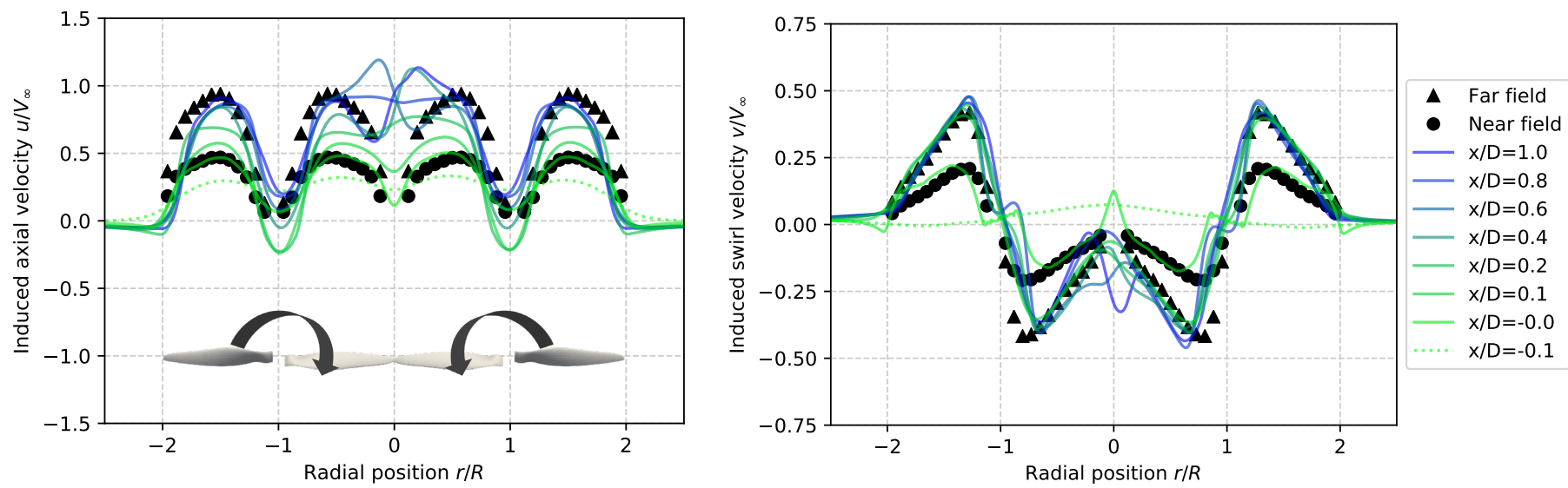

Figure 16: Comparison between momentum-theory's near/far field velocities and VPM velocities across counter-rotating multirotor wake in Figure 17, showing the ability of VPM to characterize velocities across wake mixing. 
results hint that the momentum-theory far field velocity is applicable through most of the transition region, but it is inadequate for predicting the induced velocity after vortex breakdown. This indicates a potential limitation of momentum theory in conceptual analysis especially important for small diameter propellers as used in distributed propulsion. As a reference, vortex breakdown on the APC 10x7 at a Reynolds number of $1.5 \times 10^{6}$ was observed approximately 8 diameters downstream at an advance ratio $J$ of $0.35,12$ diameters at $J=0.45$, and 20 diameters at $J=0.55$.

\section{IV.D. Multirotor Wake Mixing}

A multirotor configuration of two propellers was simulated with no separation between tips to facilitate wake mixing. We observed that the wake attains a different topology depending on whether the rotors are co-rotating or counter-rotating. In the counter-rotating case, the vortex filaments meet at the tips creating a sequence of ellipsoidal vortex rings as the wake is shed downstream. In the co-rotating case, the vortex filament meet at the tips creating a helicoidal filament that extends downstream from the plane of rotation. Since helicoids are subject to a greater vortex stretching than a closed-loop ring, the co-rotating case showed issues of numerical stability. In future work we expect these issues to be eased by implementing a scheme of particle splitting. The counter-rotating case was numerically stable and is hereby reported.

Figure 17 shows the wake developed two diameters downstream of a pair of counter-rotating APC 10x7 propellers at an advance ratio of 0.35 and Reynolds number of $1.5 \times 10^{6}$. At the plane of rotation, vortex filaments cancel each other as blade tips meet, and reconnect creating a closed-loop ring. In between the propellers it can be seen the mixing of vortextubes in a von-Kármán-like pattern further downstream (left figure). Figure 16 compares axial and swirl velocities of the counter-rotating VPM wake to the near/far field velocities of momentum theory (which ignores wake interactions). The hub of the propellers are located in the $r / R$ positions -1 and 1 . The simulation shows that both axial and swirl velocities have local peaks inboard of both propellers $(-1<r / R<1)$ that oscillate between right and left propeller in a periodic pattern as the wake is shed downstream. These unsteady periodic dynamics develop very close to the plane of rotation, which significantly affect the accuracy of a simplistic analysis tool as momentum theory, while highlighting the strength of the vortex particle method.

\section{Conclusion}

Unsteady wake dynamics of individual propellers were successfully modeled through the vortex particle method, replicating the instabilities that lead to vortex breakdown as observed experimentally. Comparing the method with results from momentum theory, it was shown that VPM is consistent with theoretical values
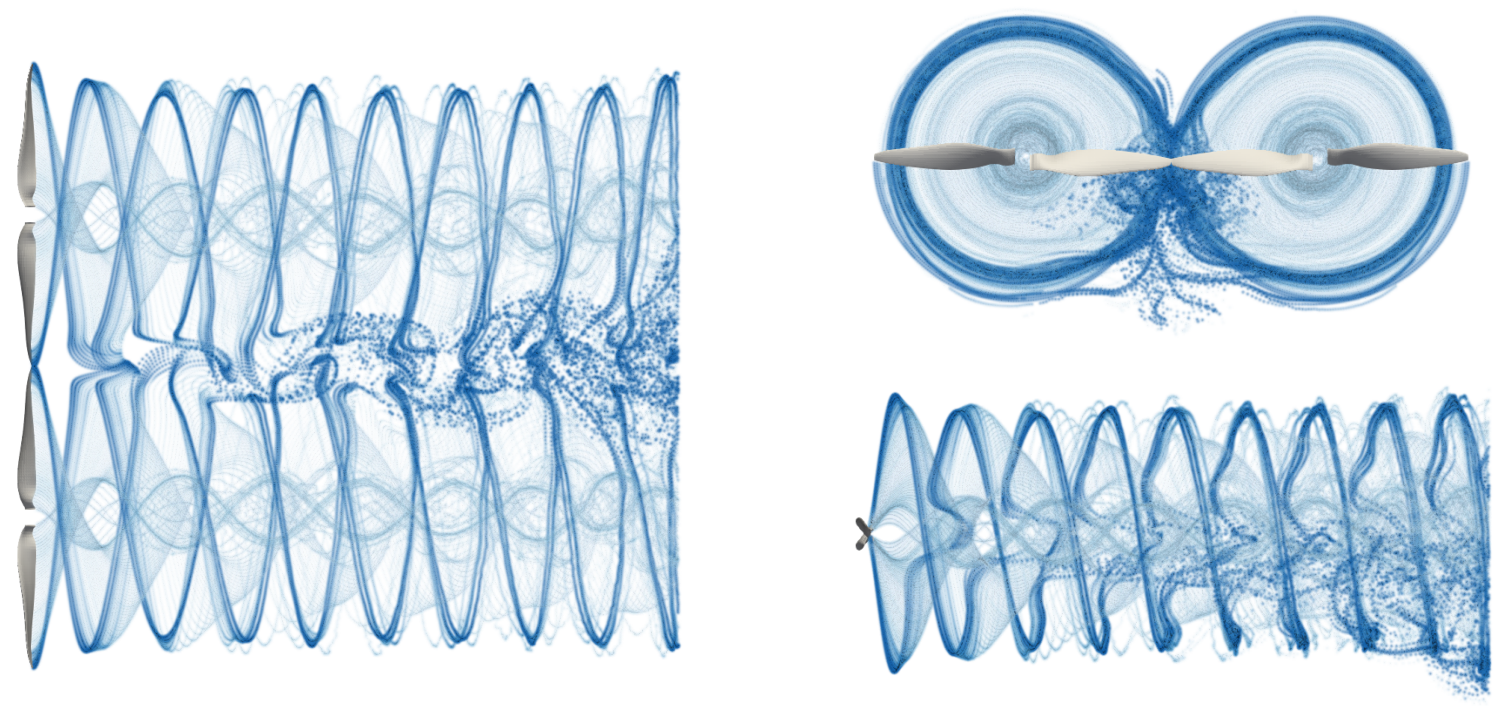

Figure 17: Wake mixing of counter-rotating APC 10x7 propellers at $J=0.35$. 
of near and far field induced velocities in the stable region, and a notable feature is its ability to model near/far field transition, which cannot be captured by momentum theory. Furthermore, VPM is able to fully characterize induced velocities across the wake, from the stable region where momentum theory operates, through transition and unstable vortex-breakdown regions where momentum theory is not applicable. VPM simulations hint that momentum theory predictions are applicable through most of the transition region, but they are inadequate for predicting induced velocities after vortex breakdown, indicating a potential limitation of momentum theory in conceptual analysis, especially important for small diameter propellers as used in distributed propulsion. The simulation of a multirotor configuration of two tip-to-tip propellers was shown, displaying the capacity of VPM to model wake mixing.

In future work, wake velocity in the unstable region and transition will be further validated quantitatively, enabling to use of VPM in analysis of prop-on-wing interaction after vortex breakdown. Also, we will close the loop between unsteady wake dynamics and load distribution, and perform a parametric study of propeller performance affected by prop-on-prop interaction. The development of this vortex particle code is still a work in progress, but the results presented here are an intermediate step towards a robust tool for the modeling of wake interaction in distributed propulsion.

\section{Acknowledgments}

This work is funded by the High Impact Doctoral Research fellowship granted by Brigham Young University.

\section{References}

${ }^{1}$ Berdowski, T. J., 3D Lagrangian VPM-FMM for Modelling the Near-Wake of a HAWT, Thesis, Technical University of Denmark and Delft University of Technology, 2015.

${ }^{2}$ Calabretta, J. S., A Three Dimensional Vortex Particle-Panel Code For Modeling Propeller-Airframe Interaction, Thesis, California Polytechnic State University, San Luis Obispo, California, jun 2010.

${ }^{3}$ Stock, M., Gharakhani, A., and Stone, C., "Modeling Rotor Wakes with a Hybrid OVERFLOW-Vortex Method on a GPU Cluster," 28th AIAA Applied Aerodynamics Conference, , No. July, 2010, pp. 1-12.

${ }^{4} \mathrm{He}$, C. and Zhao, J., "Modeling Rotor Wake Dynamics with Viscous Vortex Particle Method," AIAA Journal, Vol. 47, No. 4, apr 2009, pp. 902-915.

${ }^{5}$ Zhao, J. and He, C., "A Finite State Dynamic Wake Model Enhanced with Vortex Particle MethodDerived Modeling Parameters for Coaxial Rotor Simulation and Analysis," Journal of the American Helicopter Society, Vol. 61, No. 2, 2016.

${ }^{6} \mathrm{He}, \mathrm{C}$. and Rajmohan, N., "Modeling the Aerodynamic Interaction of Multiple Rotor Vehicles and Compound Rotorcraft with Viscous Vortex Particle Method," AHS 72nd Annual Forum, No. August, 2016.

${ }^{7}$ Ho, J. C. and Yeo, H., "Assessing Calculated Blade Loads of the Tilt Rotor Aeroacoustic Model," Journal of Aircraft, Vol. 55, No. 3, may 2018, pp. 1287-1298.

${ }^{8}$ Wang, Y., Abdel-Maksoud, M., and Song, B., "Simulating marine propellers with vortex particle method," Physics of Fluids, Vol. 29, No. 1, jan 2017, pp. 017103.

${ }^{9}$ Willis, D. J., An Unsteady, Accelerated, High Order Panel Method with Vortex Particle Wakes, Ph.D. thesis, Massachusetts Institute of Technology, 2006.

${ }^{10}$ Wang, H., Zhou, Z., Xu, X., and Zhu, X., "Influence analysis of propeller location parameters on wings using a panel/viscous vortex particle hybrid method," The Aeronautical Journal, Vol. 122, No. 1247, 2018, pp. 21-41.

${ }^{11}$ Calabretta, J. S. and Mcdonald, R. A., "A Three Dimensional Vortex Particle-Panel Method for Modeling PropulsionAirframe Interaction," 48th AIAA Aerospace Sciences Meeting Including the New Horizons Forum and Aerospace Exposition, , No. January, 2010, pp. 2010.

${ }^{12}$ Chatelain, P., Duponcheel, M., Caprace, D.-G., Marichal, Y., and Winckelmans, G., "Vortex particle-mesh simulations of vertical axis wind turbine flows: from the airfoil performance to the very far wake," Wind Energy Science, Vol. 2, No. 1, jun 2017, pp. 317-328.

${ }^{13}$ Ploumhans, P., Daeninck, G., and Winckelmans, G., "Simulation of three-dimensional bluff-body flows using the vortex particle and boundary element methods," Flow, Turbulence and Combustion, Vol. 73, No. 2, 2004, pp. 117-131.

${ }^{14}$ Willis, D., Peraire, J., and White, J., "A Combined pFFT - Multipole Tree Code, Unsteady Panel Method with Vortex Particle Wakes," 43rd AIAA Aerospace Sciences Meeting and Exhibit, No. January, American Institute of Aeronautics and Astronautics, Reston, Virigina, jan 2005.

${ }^{15}$ Winckelmans, G., Cocle, R., Dufresne, L., and Capart, R., "Vortex methods and their application to trailing wake vortex simulations," Comptes Rendus Physique, Vol. 6, No. 4-5 SPEC. ISS., 2005, pp. 467-486.

${ }^{16}$ Yokota, R. and Barba, L. A., "Comparing the treecode with FMM on GPUs for vortex particle simulations of a leapfrogging vortex ring," Computers and Fluids, Vol. 45, No. 1, 2011, pp. 155-161.

${ }^{17}$ Singh, P. and Friedmann, P. P., "Application of Vortex Methods to Coaxial Rotor Wake and Load Calculations in Hover," Journal of Aircraft, Vol. 55, No. 1, 2017, pp. 1-9.

${ }^{18}$ Tan, J., Sun, Y., and Barakos, G., "Unsteady loads for coaxial rotors in forward flight computed using a vortex particle method," Aeronautical Journal, Vol. 122, No. 1251, 2018, pp. 693-714. 
${ }^{19}$ Zhao, J. and He, C., "Enhancement of Viscous Vortex Particle Method for Fundamental Rotor Wake Dynamics Simulation," New Horizons, , No. January, 2012, pp. 1-11.

${ }^{20}$ Tan, J. F. and Wang, H. W., "Simulating unsteady aerodynamics of helicopter rotor with panel/viscous vortex particle method," Aerospace Science and Technology, Vol. 30, No. 1, 2013, pp. 255-268.

${ }^{21}$ Shi, Y., Xu, G., and Wei, P., "Rotor wake and flow analysis using a coupled EulerianLagrangian method," Engineering Applications of Computational Fluid Mechanics, Vol. 10, No. 1, jan 2016, pp. 384-402.

${ }^{22}$ Shi, Y., Xu, Y., Xu, G., and Wei, P., "A coupling VWM/CFD/CSD method for rotor airload prediction," Chinese Journal of Aeronautics, Vol. 30, No. 1, feb 2017, pp. 204-215.

${ }^{23}$ Battey, L. S., A Hybrid Navier Stokes/Vortex Particle Wake Methodology for Modeling Helicopter Rotors in Forward Flight and Maneuvers, Thesis, Georgia Institute of Technology, 2018.

${ }^{24}$ Purcell, E. M. and Morin, D. J., Electricity and Magnetism, Cambridge University Press, third edit ed., mar 2013.

${ }^{25}$ Winckelmans, G. and Leonard, A., "Contributions to Vortex Particle Methods for the Computation of Three-Dimensional Incompressible Unsteady Flows," Journal of Computational Physics, Vol. 109, No. 2, dec 1993, pp. $247-273$.

${ }^{26}$ Eldredge, J. D., Leonard, A., and Colonius, T., "A General Deterministic Treatment of Derivatives in Particle Methods," Journal of Computational Physics, Vol. 180, No. 2, aug 2002, pp. 686-709.

${ }^{27}$ Degond, P. and Mas-Gallic, S., "The Weighted Particle Method for Convection-Diffusion Equations. Part 1: The Case of an Isotropic Viscosity," Mathematics of Computation, Vol. 53, No. 188, oct 1989, pp. 485.

${ }^{28}$ Barnes, J. and Hut, P., "A hierarchical $\mathrm{O}(\mathrm{N} \log \mathrm{N})$ force-calculation algorithm," Nature, Vol. 324, No. 6096, 1986, pp. 446-449.

${ }^{29}$ Greengard, L., Rokhlin, V., Rokhlin, L. G., and V., "A fast algorithm for particle simulations," Journal of Computational Physics, Vol. 73, No. 2, 1987, pp. 325-348.

${ }^{30}$ Greengard, L. F., The Rapid Evaluation Of Potential Fields In Particle Systems, Phd thesis, Yale University, 1987.

${ }^{31}$ Cheng, H., Greengard, L., and Rokhlin, V., "A Fast Adaptive Multipole Algorithm in Three Dimensions," Journal of Computational Physics, Vol. 155, No. 2, 1999, pp. 468-498.

${ }^{32}$ Yokota, R. and Barba, L. A., "Treecode and fast multipole method for N-body simulation with CUDA," GPU Computing Gems Emerald Edition, 2011, pp. 113-132.

${ }^{33}$ Yokota, R. and Barba, L. A., "Computers \& Fluids FMM-based vortex method for simulation of isotropic turbulence on GPUs, compared with a spectral method," Computers and Fluids, Vol. 80, 2013, pp. 17-27.

${ }^{34}$ Squire, W. and Trapp, G., "Using complex variables to estimate derivatives of real functions," SIAM Review, Vol. 40, No. 1, 1998, pp. 110-112.

${ }^{35}$ Lyness, J. N., "Numerical algorithms based on the theory of complex variable," Proceedings of the 1967 22nd national conference on -, 1967, pp. 125-133.

${ }^{36}$ Fornberg, B., "Numerical Differentiation of Analytic Functions," ACM Trans. Math. Softw., Vol. 7, No. 4, 1981, pp. 512526.

${ }^{37}$ Martins, J., Kroo, I., and Alonso, J., "An automated method for sensitivity analysis using complex variables," 38th Aerospace Sciences Meeting and Exhibit, American Institute of Aeronautics and Astronautics, Reston, Virigina, jan 2000.

${ }^{38}$ Kenway, G. K. W., Kennedy, G. J., and Martins, J. R. R. A., "Scalable Parallel Approach for High-Fidelity Steady-State Aeroelastic Analysis and Adjoint Derivative Computations," AIAA Journal, Vol. 52, No. 5, may 2014, pp. $935-951$.

${ }^{39}$ Fielder, R., Montoya, A., Millwater, H., and Golden, P., "Residual stress sensitivity analysis using a complex variable finite element method," International Journal of Mechanical Sciences, Vol. 133, No. November 2016, nov 2017, pp. 112-120.

${ }^{40}$ Winckelmans, G. S., Topics in vortex methods for the computation of three and two dimensional incompressible unsteady flows, Phd thesis, California Institute of Technology, 1989.

${ }^{41}$ Pedrizzetti, G., "Insight into singular vortex flows," Fluid Dynamics Research, Vol. 10, No. 2, 1992, pp. $101-115$.

${ }^{42}$ Williamson, J. H., "Low-storage Runge-Kutta schemes," Journal of Computational Physics, Vol. 35, No. 1, 1980, pp. 4856 .

${ }^{43}$ Brandt, J. B., Deters, R. W., Ananda, G. K., and Selig, M. S., "UIUC Propeller Database," http://mselig.ae.illinois.edu/props/propDB.html.

${ }^{44}$ McCrink, M. H. and Gregory, J. W., "Blade Element Momentum Modeling of Low-Reynolds Electric Propulsion Systems," Journal of Aircraft, Vol. 54, No. 1, 2017, pp. 163-176.

${ }^{45} \mathrm{Du}$, Z. and Selig, M., "A 3-D stall-delay model for horizontal axis wind turbine performance prediction," 1998 ASME Wind Energy Symposium, American Institute of Aeronautics and Astronautics, Reston, Virigina, jan 1998.

${ }^{46}$ Viterna, L. A. and Janetzke, D. C., "Theoretical and experimental power from large horizontal-axis wind turbines," Tech. rep., Washington Procurement Operations Office, Washington, DC (United States), sep 1982.

${ }^{47}$ Ning, S. A., "A simple solution method for the blade element momentum equations with guaranteed convergence," Wind Energy, Vol. 17, No. 9, jul 2014, pp. 1327-1345.

${ }^{48}$ Bertin, J. J. and Cummings, R. M., Aerodynamics for Engineers.

${ }^{49}$ Saffman, P. G., "Dynamics of vorticity," Journal of Fluid Mechanics, Vol. 106, No. -1, 1981, pp. 49.

${ }^{50}$ Felli, M., Camussi, R., and Di Felice, F., "Mechanisms of evolution of the propeller wake in the transition and far fields," Journal of Fluid Mechanics, Vol. 682, 2011, pp. 5-53.

${ }^{51}$ Sullivan, I. S., Niemela, J. J., Hershberger, R. E., Bolster, D., and Donnelly, R. J., "Dynamics of thin vortex rings," Journal of Fluid Mechanics, Vol. 609, aug 2008, pp. 319-347.

${ }^{52}$ Konstantinov, M., "Numerical investigation of the interaction of coaxial vortex rings," International Journal of Numerical Methods for Heat \& Fluid Flow, Vol. 7, No. 2/3, mar 1997, pp. 120-140.

${ }^{53}$ Kleckner, D. and Irvine, W. T. M., "Creation and dynamics of knotted vortices," Nature Physics, Vol. 9, No. 4, 2013, pp. 253-258. 
${ }^{54}$ Quaranta, H. U., Bolnot, H., and Leweke, T., "Long-wave instability of a helical vortex," Journal of Fluid Mechanics, Vol. 780, 2015, pp. 687-716.

${ }^{55}$ Leishman, D. P. F. J. G., Principles of Helicopter Aerodynamics, 2006.

${ }^{56}$ Lee, D. J. and Na, S. U., "Numerical simulations of wake structure generated by rotating blades using a time marching, free vortex blob method," European Journal of Mechanics - B/Fluids, Vol. 18, No. 1, 1999, pp. 147-159.

${ }^{57}$ Landgrebe, A. J., "The wake geometry of a hovering helicopter rotor an its influence on rotor performance," Journal of the American Helicopter Society, Vol. 17, No. 4, 1972, pp. 3-15.

${ }^{58}$ Burton, T., Jenkins, N., Sharpe, D., and Bossanyi, E., Wind Energy Handbook, 2011. 